\title{
Graphs, hypergraphs, and properads
}

\author{
JOACHIM KOCK ${ }^{1}$ \\ kock@mat.uab.cat
}

\begin{abstract}
A categorical formalism for directed graphs is introduced, featuring natural notions of morphisms and subgraphs, and leading to two elementary descriptions of the free-properad monad, first in terms of presheaves on elementary graphs, second in terms of groupoid-enriched hypergraphs.
\end{abstract}

\section{Contents}

\begin{tabular}{|c|c|c|}
\hline \multicolumn{3}{|c|}{ Introduction } \\
\hline \multirow[t]{7}{*}{1} & Gra & phs \\
\hline & 1.1 & Graphs \\
\hline & 1.2 & Connectedness and acyclicity \\
\hline & 1.3 & Closed-graph adjunction \\
\hline & 1.4 & Canonical neighbourhoods, covers and hulls \\
\hline & 1.5 & Pushouts, coequalisers, and colimits over graphs \\
\hline & & Complements and convexity \\
\hline \multirow[t]{5}{*}{2} & Pro & perads \\
\hline & 2.1 & Digraphical species (coloured bi-collections) and F-graphs \\
\hline & 2.2 & The free-properad monad $\ldots \ldots$ \\
\hline & 2.3 & Generic/free factorisation and nerve theorem . \\
\hline & 2.4 & Working in the category $\widetilde{G} r . \ldots \ldots$ \\
\hline \multirow[t]{5}{*}{3} & Hy & ergraphs \\
\hline & 3.1 & Discrete hypergraphs \\
\hline & 3.2 & Groupoid-enriched hypergraphs \\
\hline & 3.3 & The free properad on a groupoid-valued bi-collection \\
\hline & 3.4 & Free-properad monad on the category of hypergraphs \\
\hline
\end{tabular}

\section{Introduction}

Properads were introduced by Vallette [17], as a notion intermediate between operads and props, featuring important notions and results generalised from operads, such as Koszul duality. From a combinatorial viewpoint, properads are to connected acyclic directed graphs (henceforth just called graphs) as operads are to rooted trees.

A combinatorial approach to coloured properads and infinity-properads has been developed by Hackney, Robertson and Yau [10], based on a somewhat elaborate notion

\footnotetext{
${ }^{1}$ Departament de Matemàtiques, Universitat Autònoma de Barcelona, Spain
} 
of graph (due to Yau and Johnson [20]), whose notions of morphism and subgraph are derived from properad notions, in turn defined in terms of an operation of graph substitution (also from [20]).

The present work (which grew out of studying [10]) proposes a different approach to the relationship between graphs and properads, in which the starting point is a graph formalism featuring natural notions of morphisms and subgraphs, and featuring colimits enough to describe the free-properad monad in terms of presheaves on elementary (directed) graphs, and to prove a nerve theorem, in close analogy with the approach to graphs and coloured modular operads (compact symmetric multicategories) of Joyal-Kock [11]. A second feature of the graph formalism introduced is that it naturally extends to hypergraphs, and neatly explains the dual role of graphs as carriers of algebraic structures (3.1.5).

The theory is developed from scratch (finite sets, pullbacks, colimits), and follows the case of operads [13] as closely as possible.

In the case of operads, there is a natural category of trees and tree embeddings [13], with a subcategory of elementary trees, such that (coloured) collections (the structure underlying coloured operads) are precisely presheaves on elementary trees, or equivalently sheaves on trees. The free-operad monad is given by a simple colimit formula exploiting this equivalence of categories. The free operad on a tree is not again a tree, but one discovery of [13] is that nevertheless it is represented by the same shape

$$
A \leftarrow E \rightarrow B \rightarrow A \text {. }
$$

This shape is that of polynomial endofunctors, and the free-operad monad restricts to the free-monad monad on polynomial endofunctors, where it has a direct combinatorial description in terms of these representing diagrams [13].

The same features are shared by the case of properads: a natural category $\mathbf{G r}$ of (connected, acyclic, directed) graphs is introduced, with a subcategory elGr of elementary graphs, such that (coloured) bi-collections (the structure underlying coloured properads) are precisely presheaves on elGr, or equivalently sheaves on Gr. Again the freeproperad monad is given by a simple colimit formula exploiting this equivalence of categories. This time, however, the category of graphs Gr involves etale maps instead of just embeddings, and the notion of sheaves is with respect to the etale topology. This is a crucial difference: in contrast to embeddings, etale maps have automorphisms (deck transformations), and for this reason, when trying to mimic the representability feature, groupoids are required, instead of sets. With this proviso, the analogy from trees goes through: while the free properad on a graph is not again a graph, it is a diagram of the same shape (now in groupoids), and this shape,

$$
A \leftarrow I \rightarrow N \leftarrow O \rightarrow A
$$

is that of (groupoid-enriched) hypergraphs (hypergraphs with 'stacky' nodes). Again, the free-properad monad restricts to a monad on such hypergraphs, and has a direct combinatorial interpretation in terms of these representing diagrams: while a hypergraph is given by its elementary subgraphs (or more precisely, by etale maps from elementary graphs), the free properad on it is given by etale maps from arbitrary (connected) graphs. 
In summary, the main notions involved fit into the following schematic relationship:

\begin{tabular}{|c|c|c|c|c|}
\hline elementary tree & tree & polynomial endo. & presheaf on elem. trees & operad \\
\hline elementary graph & graph & hypergraph & presheaf on elem. graphs & properad \\
\hline
\end{tabular}

The category Gr encodes 'geometric' aspects of the combinatorics of graphs - open inclusions, etale maps, symmetries, colimits. Again in analogy with the case of operads and trees, the free-properad monad generates a bigger category of graphs $\widetilde{\mathbf{G} r}$, whose new maps, the graph refinements, embody 'algebraic' aspects - basically substitution (see 2.3). This bigger category $\widetilde{G} r$ is featured in a nerve theorem (2.3.9), characterising properads among presheaves on $\widetilde{G r}$ in terms of a Segal condition. The category $\widetilde{G r}$ is shown to have a weak factorisation system given by refinements and etale maps. Cutting down the right-hand class from etale maps to convex open inclusions results in a smaller category, which is the one first constructed by Hackney, Robertson and Yau [10].

Some of the results in Subsections 2.2 and 2.4 have some overlap with results in Hackney-Robertson-Yau [10], as indicated in each case. The reader is strongly encouraged to follow these references to compare with a different approach with its own advantages.

\section{Graphs}

\subsection{Graphs}

In this work, the word 'graph' means 'directed graph with open-ended edges' (and from Section 2 and onwards, graphs will be assumed connected and acyclic). We proceed to give the formal definition, whose merit is the elegant way morphisms and subgraphs are encoded. All constructions take place in the category of finite sets. When numbers are used as sets, they denote a set with that many elements.

1.1.1 Definition. A graph is a diagram of finite sets

$$
A \stackrel{s}{\longleftarrow} I \stackrel{p}{\longrightarrow} N \stackrel{q}{\longleftarrow} O \stackrel{t}{\longrightarrow} A
$$

for which $s$ and $t$ are injective.

Throughout, for any individual graph under consideration, we shall use these symbols to refer to its constituents, if no confusion seems likely.

1.1.2 Terminology and interpretation. The set $A$ is the set of edges (' $A$ ' for 'arc' or 'arête'). The set $N$ is the set of nodes. The set $I$ is the set of in-flags, and the set $O$ is the set of out-flags. The maps $s$ and $t$ return the edge in a flag, and the maps $p$ and $q$ return the node in a flag. Saying that $s$ is injective means that every edge is the incoming edge of at most one node, and similarly injectivity of $t$ means that every edge is the outgoing edge of at most one node. 
An edge $a \in A$ is called an inner edge if it belongs to the intersection $O \cap I=$ $O \times{ }_{A} I \subset A$. The complement is called the set of ports. The complement of $s$, i.e. the set of edges which are not incoming edges of any node, is called the set of exports. The complement of $t$ is called the set of imports.

1.1.3 Unit graph. The graph with one edge and without nodes is given by

$$
1 \longleftarrow 0 \longrightarrow 0 \longleftarrow 0 \longrightarrow 1 .
$$

This edge is simultaneously an import and an export (indeed the unique edge is neither in the image of $s$, nor in the image of $t)$. It is called the unit graph, denoted $U$.

Note that the edge itself has no sense of direction. The notion of direction in a graph is provided only by the nodes, owing to the distinction made between in-flags and out-flags. (Compare with categories: an object has no sense of direction; arrows (operations) do have a direction, expressed by source and target. Further explanation of this analogy is provided by the ambient category of hypergraphs, cf. 3.1 .5 below.)

1.1.4 Corollas. The corolla with $m$ imports and $n$ exports, denoted $C_{n}^{m}$, is the graph with one node given by

$$
m+n \longleftarrow m \longrightarrow 1 \longleftarrow n \longrightarrow m+n,
$$

with the outer maps the obvious sum inclusions. As a special case we have the graph $C_{0}^{0}$ with one node and no edges, given by

$$
0 \longleftarrow 0 \longrightarrow 1 \longleftarrow 0 \longrightarrow 0 .
$$

1.1.5 Wheels. The graph $W_{1}$ with one node and one 'loop' (cf. 1.2.6) is given by

$$
1 \longleftarrow 1 \longrightarrow 1 \longleftarrow 1 \longrightarrow 1 \text {. }
$$

More generally, the wheel of length $n \geq 1$, is the graph denoted $W_{n}$ given by

$$
n \stackrel{s}{\longleftarrow} n \stackrel{p}{\longrightarrow} n \stackrel{q}{\longleftarrow} n \stackrel{t}{\longrightarrow} n,
$$

for which all the structure maps $s, t, p, q$ are bijections, and such that the composite bijection $t \circ q^{-1} \circ p \circ s^{-1}: n \stackrel{\sim}{\rightarrow} n$ is a cyclic permutation of $n$.

1.1.6 Philosophical remarks. From the viewpoint of properads, the nodes in a graph represent operations with multiple in- and outputs. From this viewpoint, it is natural to try to define graphs as pairs of multi-valued maps

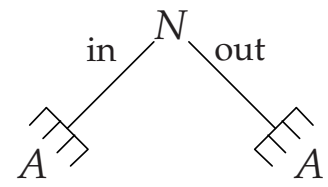


A standard way to encode a multi-valued map is as a span. Hence we arrive at the shape (1).

On the other hand, since a closed directed graph is an endospan $E \rightrightarrows V$ (source and target of an edge), a directed graph admitting open-ended edges should be the same but with just partially defined maps. A standard way to encode a partially defined map is as a span in which the backward arrow is an injection, hence again we arrive at our shape of diagrams for a graph.

These dual viewpoints also point towards the natural relationship with hypergraphs: the shape is naturally the juxtaposition of the incidence relations a-hyperedgebeing-the-input-of-a-node and a-hyperedge-being-the-output-of-a-node, which is one way to represent directed hypergraphs, cf. 3.1.1 below.

A main feature and motivation for the present graph implementation are the elegant notions of morphisms that follow from the definition.

1.1.7 Morphisms. A morphism of graphs is a commutative diagram

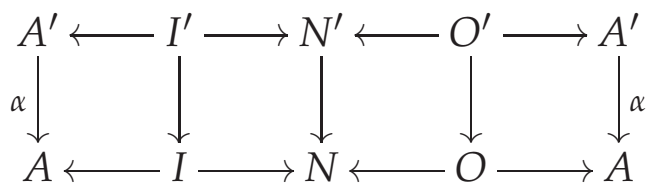

Graphs and morphisms of graphs form a category denoted $\mathrm{Gr}^{+}$.

Note that a morphism sends edges to edges and nodes to nodes, respecting the incidence relations. In particular it sends inner edges to inner edges. Ports are not necessarily sent to ports: a port may be sent to an inner edge.

A morphism is etale if the two squares in the middle are pullbacks:

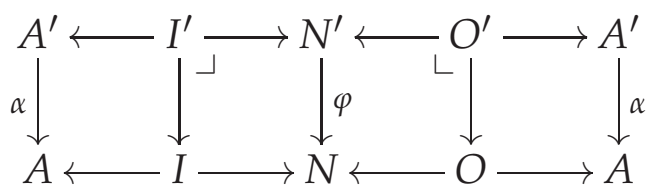

Equivalently, for each node $x \in N^{\prime}$, we have bijections $I_{x}^{\prime} \simeq I_{\varphi x}$ and $O_{x}^{\prime} \simeq O_{\varphi x}$, the subscripts indicating fibres. Hence etale means arity preserving. Denote by $\mathbf{G r}_{\text {et }}^{+}$the category of graphs and etale maps. (The notion of etale map has a clear intuitive content. It also fits into the axiomatic notion of classes of etale maps of Joyal-Moerdijk [12].)

A graph inclusion is a morphism which is levelwise injective. A subgraph of a graph $G$ is an equivalence class of graph inclusions into $G$. An open subgraph is a subgraph whose inclusion is etale.

1.1.8 Example. The unique map $C_{1}^{1} \rightarrow W_{1}$,

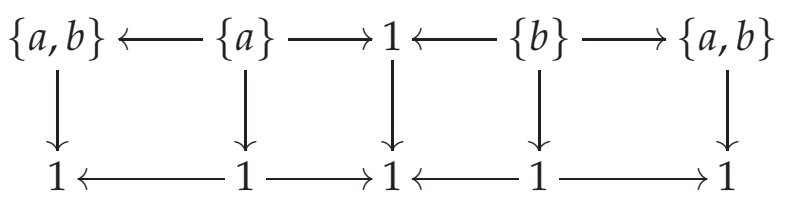

is etale but not a graph inclusion. 
1.1.9 Port-preserving maps. The inclusion of an edge is a diagram

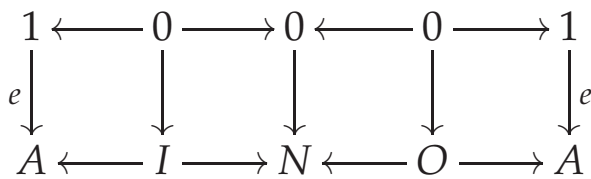

It is an import precisely when the right-most square is a pullback, and it is an export precisely when the left-most square is a pullback. Correspondingly, a morphism of graphs is called import preserving if the right-most square is a pullback, and export preserving if the left-most square is a pullback.

1.1.10 Isolated nodes. A node $x$ in a graph is called isolated when both $I_{x}$ and $O_{x}$ are empty.

1.1.11 Proposition. Except in the case where $G^{\prime}$ has an isolated node, a morphism of graphs $G^{\prime} \rightarrow G$ is completely determined by its values on edges. Precisely, the natural map $\operatorname{Hom}_{\mathbf{G r}^{+}}\left(G^{\prime}, G\right) \rightarrow \operatorname{Hom}_{\text {Set }}\left(A^{\prime}, A\right)$ is injective.

Proof. The injectivity axiom implies that $A^{\prime} \rightarrow A$ determines also $I^{\prime} \rightarrow I$ and $O^{\prime} \rightarrow O$. If $x \in N^{\prime}$ is a node in $G^{\prime}$, since it is assumed not to be isolated, it must be the image of some flag, either in $I^{\prime}$ or in $O^{\prime}$. In either case its image is forced by the image of that flag.

1.1.12 Relation with general graphs in the sense of Joyal-Kock. Recall that according to [11] a Feynman graph is a diagram of finite sets

$$
{ }_{i} \mathrm{C} \stackrel{s}{\longleftarrow} H \stackrel{t}{\longrightarrow} V
$$

such that $s$ is injective and $i$ is a fixpoint-free involution.

The data of a directed graph in the sense of 1.1.1 can equivalently be presented as

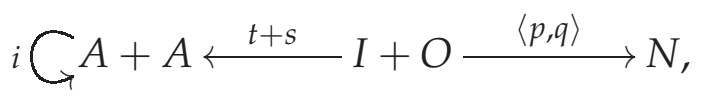

where $i$ is the natural involution on $A+A$. Hence a directed graph has an underlying Feynman graph. This is easily seen to be the object part of a faithful functor from directed graphs (and etale maps) to Feynman graphs. In fact, directed graphs in the sense of 1.1.1 are precisely $D$-graphs for a certain graphical species $D$, in the sense of [11].

For further comparison between different implementations of the notion of graph, see [1].

\subsection{Connectedness and acyclicity}

We shall be concerned mostly with connected acyclic graphs. We proceed to introduce these notions. 
1.2.1 Sums. The category $\mathbf{G r}^{+}$(as well as the subcategory $\mathbf{G r}_{\mathrm{et}}^{+}$) has categorical sums, and the empty graph

$$
0 \longleftarrow 0 \longrightarrow 0 \longleftarrow 0 \longrightarrow 0 .
$$

as initial object. Sums are calculated level-wise. They amount to disjoint union of graphs.

1.2.2 Connectedness. Recall that $W_{1}$ is the graph $1=1=1=1=1$ with one node and one loop (in fact the terminal object in $\mathbf{G r}^{+}$(but not in $\mathbf{G r}_{\mathrm{et}}^{+}$)). A graph $\mathrm{X}$ is connected if

$$
\operatorname{Hom}\left(X, W_{1}+W_{1}\right)=2 \text {. }
$$

In other words, $X$ is non-empty and every morphism to $W_{1}+W_{1}$ is constant. Equivalently, $X$ is non-empty and is not a sum of smaller graphs. (Equivalently, in its most categorical formulation, a graph $X$ is connected when $\operatorname{Hom}(X,-)$ preserves finite sums.)

1.2.3 Example. A graph for which all the structure maps are bijections is precisely a disjoint union of wheels. In fact the full subcategory spanned by graphs of this type is equivalent to the category of finite-sets-with-a-permutation (by cycle-decomposition of permutations).

1.2.4 Acyclicity. A graph $X$ is called acyclic (or wheel-free) if

$$
\operatorname{Hom}\left(W_{n}, X\right)=0, \quad \forall n>0 .
$$

In other words, $X$ does not admit a morphism from any wheel, or equivalently, does not contain a wheel as a subgraph.

1.2.5 Trees and linear graphs. An acyclic graph is a forest when $q$ is a bijection. An acyclic graph is a tree when $q$ is a bijection and there is a unique export (compare [13]). It is a linear tree (or linear graph) if both $p$ and $q$ are bijections, and there is a unique export. Denote by $L_{k}$ the linear graph with $k$ nodes.

1.2.6 Loops. A loop is an edge which is simultaneously an input and an output for the same node. In other words, $a \in A$ is a loop if there is a node $x$ such that $a \in I_{x} \cap O_{x}$. Equivalently, a loop in $X$ is the image edge of a map $W_{1} \rightarrow X$. Accordingly, a node is loopfree if $I_{x}+O_{x} \rightarrow A$ is injective. A graph is loopfree if every node is loopfree. From the $W_{1}$-characterisation of loops, it is clear that an acyclic graph is loopfree.

\subsection{Closed-graph adjunction}

1.3.1 Closed graphs. A closed graph is a directed graph in the classical sense, i.e. a presheaf on $\bullet \leftleftarrows \bullet$. We use the standard letters

$$
E \stackrel{s}{\stackrel{s}{\leftrightarrows}} V .
$$


To a closed graph is associated a graph in the sense of 1.1.1, namely

$$
E \stackrel{=}{\longleftarrow} \stackrel{t}{\longrightarrow} V \stackrel{s}{\longleftarrow} E \stackrel{=}{\longrightarrow} E .
$$

This defines a fully faithful functor from closed graphs to graphs. Its essential image is the subcategory of graphs for which the end maps are bijections. We also call these closed graphs.

1.3.2 The core of a graph. The core of a graph $X$ is the closed graph $O \times_{A} I \rightrightarrows N$ given by the diagram

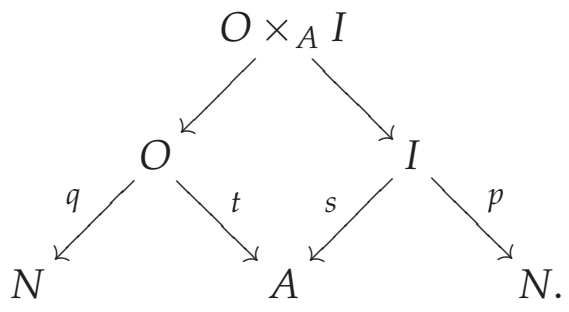

It is denoted $X^{\bullet}$. Viewed as a graph, the core is represented as follows, together with its canonical map to $X$ :

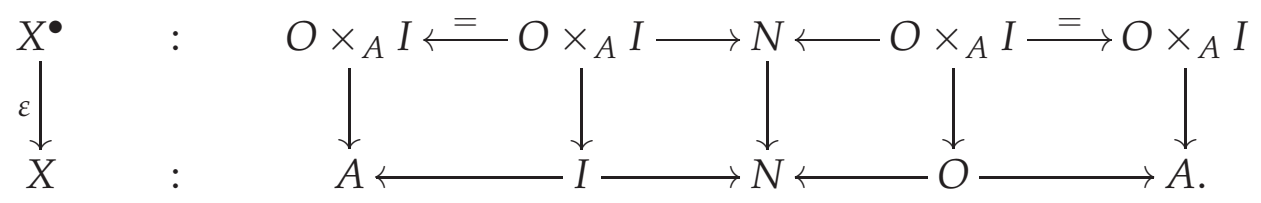

Taking core amounts to deleting all ports (i.e. replacing the set $A$ by the subset $O \times{ }_{A} I$ of inner edges), hence $X^{\bullet}$ is a subgraph of $X$. It is clear that taking core is functorial, and it follows easily from the universal property of the pullback that

1.3.3 Proposition. Taking core is right adjoint to the inclusion of closed graphs into graphs. The counit is $\varepsilon$.

1.3.4 Core equivalences. A graph map $f: Y \rightarrow X$ is called a core equivalence if $f^{\bullet}$ : $Y^{\bullet} \rightarrow X^{\bullet}$ is invertible.

1.3.5 Lemma. The etale maps are precisely the maps right orthogonal to the class of core equivalences between connected graphs, or equivalently, right orthogonal to the class of all maps between corollas. The inclusions are precisely the maps right orthogonal to both $U+U \rightarrow U$ and $C_{0}^{0}+C_{0}^{0} \rightarrow C_{0}^{0}$.

\subsection{Canonical neighbourhoods, covers and hulls}

1.4.1 Canonical cover. Given a subset of nodes $N^{\prime} \subset N$ of a graph $X$, construct a new graph as

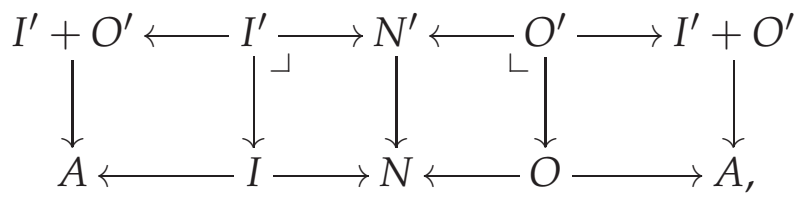


clearly a disjoint union of corollas. Jointly they cover the nodes in $N^{\prime}$. When $N^{\prime}=N$, this is called the canonical etale cover of $X$, denoted cancov $(X)$. (It is an open cover iff $X$ is loopfree.) When $N^{\prime}$ consists of a single node $x \in N$, the construction gives the canonical neighbourhood of $x$, an open subgraph when $x$ is loopfree.

1.4.2 Open hull. In the same situation, the open hull of $N^{\prime}$ is defined by gluing the edges of the corollas according to their incidences in $X$, to obtain an open subgraph in $X$. The notion of gluing will be formalised below. In the present situation, it means taking union inside $A$ instead of disjoint union: simply take the image factorisation of $I^{\prime}+O^{\prime} \rightarrow A$. Note that this includes also any existing loops at the nodes. By the universal property of union, it is the smallest open subgraph having $N^{\prime}$ as set of nodes.

1.4.3 Etale hull. Slightly more involved is the construction of the etale hull of a subgraph. In this situation we are given a subgraph $G \subset X$, and we want to factor the inclusion as a core equivalence followed by an etale map. The construction of flags is as before (forced by the etale requirement). It remains to construct the correct edge set: it is a certain pushout, over the set of inner edges of $G$. It will be important to consider a slightly more general situation. A map of graphs $X^{\prime} \rightarrow X$ is called locally injective when for each $x \in N^{\prime}$ we have that $I_{x}^{\prime} \rightarrow I_{\varphi x}$ and $O_{x}^{\prime} \rightarrow O_{\varphi x}$ are injective.

1.4.4 Proposition. Given a locally injective map of graphs $f: X^{\prime} \rightarrow X$, there exists a factorisation

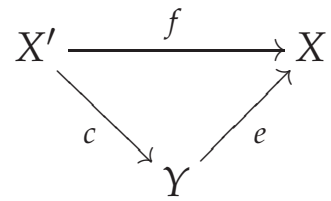

where $c$ is a core equivalence and $e$ is etale. Among these factorisations, there is an essentially unique one for which $c$ is furthermore bijective on unit components.

Proof. Given
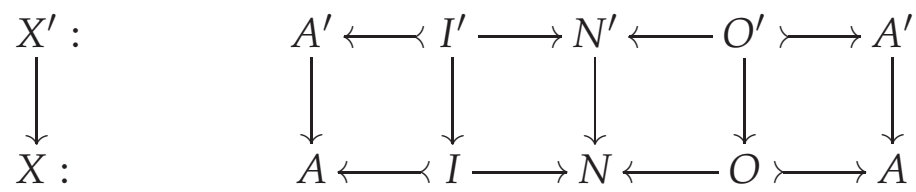

we first take the following pullbacks:

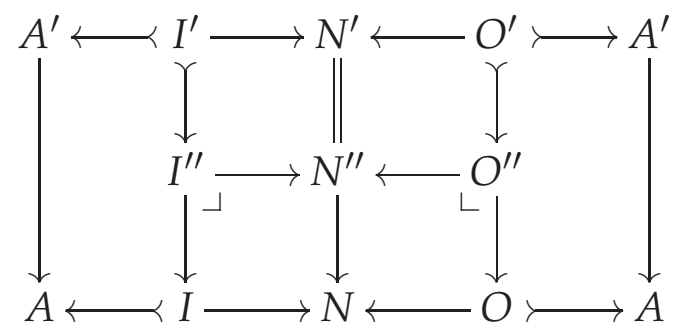

(the vertical injections by local injectivity). These choices of $I^{\prime \prime}, N^{\prime \prime}$ and $O^{\prime \prime}$ are forced by the requirement that the first map be bijcetive on nodes and the second etale. It 
remains to see if we can construct the edge set $A^{\prime \prime}$. The set $E^{\prime \prime}$ of inner edges of $Y$ must be $E^{\prime}=O^{\prime} \times{ }_{A^{\prime}} I^{\prime}$, the set of inner edges of $X^{\prime}$. The minimal choice of $A^{\prime \prime}$ to achieve this is the pushout

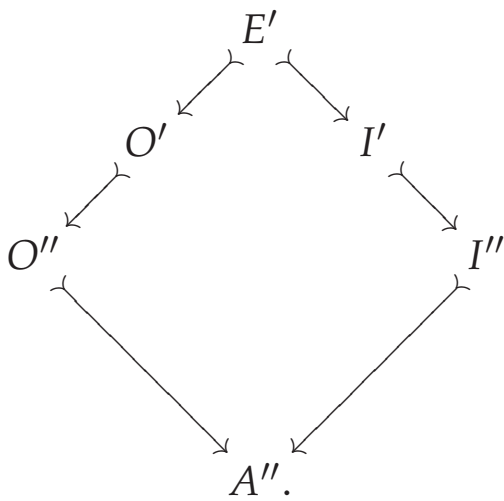

(This is also a pullback, since the maps are injective.) This choice amounts to giving $Y$ no isolated edges. This works if also $A^{\prime}$ is given as a pushout over $E^{\prime}$ (which amounts to saying that already $X^{\prime}$ had no isolated edges): in this case the map $A^{\prime} \rightarrow A$ factors uniquely through $A^{\prime \prime}$ by the universal property of the pushout. Otherwise, if $X^{\prime}$ has isolated edges, we need to add the same number of isolated edges to $Y$, that is, to add the same number of elements to $A^{\prime \prime}$. Hence the extra requirement that the map $c$ should be bijective on unit edges hence fixes the choice of $A^{\prime \prime}$ uniquely.

\subsection{Pushouts, coequalisers, and colimits over graphs}

1.5.1 Gluing data. A shrub is a disjoint union of unit graphs, i.e. a graph of the form

$$
S \longleftarrow 0 \longrightarrow 0 \longleftarrow 0 \longrightarrow S,
$$

where $S$ is a finite set. A gluing datum of a graph $G$ consist of a shrub $S$ with two injections into $G$,

$$
S \underset{\mathrm{im}}{\stackrel{\mathrm{ex}}{\rightleftarrows}} G,
$$

one export preserving, the other import preserving.

1.5.2 Proposition. The category of graphs $\mathrm{Gr}^{+}$admits coequalisers of gluing data $S \rightrightarrows G$. The quotient map $G \rightarrow Q$ is etale and bijective on nodes.

Proof. The coequaliser exists in the category of diagrams of shape (1) (i.e without imposing the injectivity condition). We just need to check the injectivity condition, i.e. that $I+I^{\prime} \rightarrow A+{ }_{S} A$ is injective (and similarly for $O$ ). But this is clear: to say that $e \in S$ maps to an export in $A^{\prime}$ means it is not in the image of $s^{\prime}$. Hence the collapse $A+A^{\prime} \rightarrow A+{ }_{S} A^{\prime}$ does not interfere with the injectivity of $I+I^{\prime} \rightarrow A+A^{\prime}$. Since the levelwise construction is just disjoint union at the level of nodes and flags, it is clear that the quotient map is bijective on nodes and etale. 
1.5.3 Corollary. The category $\mathbf{G r}_{\mathrm{et}}^{+}$of graphs and etale maps admits coequalisers of gluing data.

The colimit of a gluing datum $S \rightrightarrows G$ is constructed by connecting exports to imports in $G$, realising one connection for each unit graph in $S$. Although it is a trivial observation, it will be important to note that this colimit can be computed in steps by realising the connections one by one in any order.

1.5.4 Elementary graphs. An elementary graph is a connected graph without inner edges. Up to isomorphism there are only the following: the unit graph (one edge, no nodes), and the $(m, n)$-corollas. Let $\mathbf{e l G r} \subset \mathbf{G r}_{\text {et }}$ denote the full subcategory spanned by the elementary graphs (and all etale maps). Hence the only maps are the inclusions of an edge into a corolla, and the permutations of imports or exports.

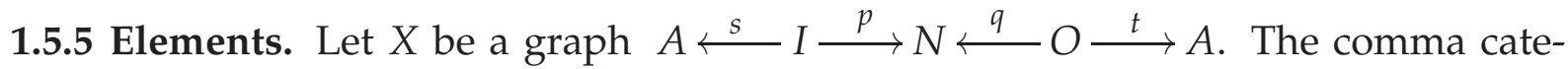
gory el $(X):=$ elGr $\downarrow X$ is called the category of elements of $X$. (See [15], Ch.II, §6, for the notion of comma category.) It has the following explicit description. Its object set is $A+N$. Its set of non-identity arrows is $I+O$. An element $f \in I$ has domain $s(f)$ and codomain $p(f)$; an element $g \in O$ has domain $t(g)$ and codomain $q(g)$. Since every arrow goes from an object in $A$ to an object in $N$, the category is really just a bipartite graph (with identity arrows added), namely the barycentric subdivision of $X$.

The category of elements el $(X)$ indexes canonically a diagram of elementary graphs, $\mathrm{el}(X) \rightarrow \mathbf{G r}^{+}$, and $X$ is the colimit of this diagram in $\mathbf{G r}^{+}$:

$$
X=\underset{E \in \operatorname{el}(X)}{\operatorname{colim}} E=\operatorname{colim}\left(\mathrm{el}(X) \rightarrow \mathbf{G r}^{+}\right) .
$$

Assuming that $X$ has no unit components, the outer edges play no role in the colimit: it can be computed equally well indexed over $\mathrm{el}\left(X^{\bullet}\right) \subset \mathrm{el}(X)$ (although of course the ingredient elementary graphs are those of $X$, not those of $\left.X^{\bullet}\right)$. The colimit indexed over $\mathrm{el}\left(\mathrm{X}^{\bullet}\right)$ is that of a gluing datum: it is the coequaliser of the canonical cover (the disjoint union of all the nodes in $X$ considered as corollas, cf. 1.4.1) over the inner edges:

$$
O \times_{A} I \rightrightarrows \operatorname{cancov}(X) \rightarrow X
$$

More generally, we have:

1.5.6 Lemma. For $X$ a graph without unit components, the functor $\operatorname{el}\left(X^{\bullet}\right) \rightarrow \operatorname{el}(X)$ is final.

1.5.7 Corollary. If $X \rightarrow Y$ is a core equivalence between graphs without unit components, then $\mathrm{el}(X) \rightarrow \operatorname{el}(Y)$ is final.

See [15], Ch.IX, §3, for the notion of final functor. What this amounts to is that if $f$ : $\mathrm{el}(X) \rightarrow \mathscr{C}$ is a functor, and if $\operatorname{el}\left(X^{\bullet}\right) \rightarrow \operatorname{el}(X) \rightarrow \mathscr{C}$ admits a colimit, then so does $f$, and the two colimits agree. 
1.5.8 Gluing datum from a graph of graphs. The case of interest in the previous discussion is the following. A functor $\mathrm{el}(X) \rightarrow \mathbf{G r}^{+}$is called a graph of graphs if it sends all $A$-objects to unit graphs, and all $I$-maps to import-preserving maps and all $O$-maps to export-preserving maps. In this case (unless $X$ is has a unit component) the restriction to $\mathrm{el}\left(X^{\bullet}\right)$ is a gluing datum, and hence has a colimit. Therefore $\mathrm{el}(X) \rightarrow \mathbf{G r}$ has a colimit. This is just the formal expression of the idea that if a graph $X$ is decorated with graphs at the nodes, then the decorating graphs (one for each node) can be glued together as prescribed by the incidence relations in the indexing graph. (In the literature, this situation (subject to a further compatibility condition, see 2.3.2) is often referred to as graph substitution (see for example [1] or [20]): the colimit is interpreted as the result of substituting each decorating graph into the corresponding node of the indexing graph.)

1.5.9 Colimit formula for etale hull. If $H \rightarrow G$ is a subgraph, then the etale hull (1.4.3) is given by

$$
\operatorname{colim}\left(\mathrm{el}(H) \rightarrow \mathrm{el}(G) \rightarrow \mathbf{G r}^{+}\right) .
$$

This just says that the etale hull is obtained by gluing together corollas from $G$ according to the shape of $H$.

1.5.10 Residue. Denote by Cor the full subcategory of $\mathbf{G r}_{\text {et }}$ consisting of the corollas. Clearly Cor is a groupoid. The residue of a connected graph $G$ is the corolla having the same imports and exports as G. This defines a functor res : $\mathbf{G r}_{\text {iso }} \rightarrow \mathbf{C o r}$ (functorial in isomorphisms, not in general maps). Note that $\operatorname{res}(U)=C_{1}^{1}$. If $x$ is a node of a graph, we shall also write res $(x)$ for the residue of the canonical neighbourhood of $x$.

1.5.11 Indexing graph for a gluing datum. A coequaliser of a gluing datum $S \rightrightarrows G$ can be interpreted as a colimit of connected graphs as follows. Write $S$ and $G$ as sums of connected components

$$
\sum_{i} U_{i} \rightrightarrows \sum_{k} G_{k}
$$

Form the graph $\sum R_{k}$ by replacing each $G_{k}$ with its residue $R_{k}=\operatorname{res}\left(G_{k}\right)$. We still have the maps from the $S$ edges into these corollas, and we can take the colimit $R$, which we call the indexing graph. The original diagram is now over $\operatorname{el}\left(R^{\bullet}\right)$, the category of elements of $R^{\bullet}$.

1.5.12 Lemma. The coequaliser $Q$ is connected if and only if the indexing graph $R$ is connected. Furthermore, in this situation, $\operatorname{res}(Q) \simeq \operatorname{res}(R)$.

Proof. To give a map $Q \rightarrow W_{1}+W_{1}$ is the same as giving a cocone $S \rightrightarrows \sum G_{k} \rightarrow W_{1}+$ $W_{1}$. Since each of the $G_{k}$ is connected, each map $G_{k} \rightarrow W_{1}+W_{1}$ is constant, and hence amounts to giving a cocone $S \rightrightarrows \sum R_{k} \rightarrow W_{1}+W_{1}$, and hence a map $R \rightarrow W_{1}+W_{1}$. Hence $Q$ is connected if and only if $R$ is. The second statement follows easily since by construction $\sum G_{k}$ and $\sum R_{k}$ have the same set of imports, of which the same subset $S$ is spent with gluing, so that also $Q$ and $R$ are left with the same set of imports. Ditto with exports. 
1.5.13 Lemma. If all the individual graphs $G_{k}$ are acyclic, and if the indexing graph $R$ is acyclic then the coequaliser $Q$ is acyclic too.

Proof. Let $W \rightarrow Q$ be a wheel in $Q$. For each of the connected graphs $G_{k}$, consider the pullback

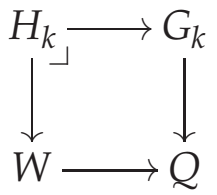

Since we have assumed $G_{k}$ acyclic, each $H_{k}$ is a sum of linear graphs, and each $H_{k} \rightarrow G_{k}$ preserves imports and exports. This means that it makes sense to take residue of each $H_{k} \rightarrow G_{k}$, yielding in each case a map $T_{k} \rightarrow R_{k}=\operatorname{res}\left(G_{k}\right)$, where each $T_{k}$ is a sum of $L_{1}$-graphs. These linear graphs $L_{1}$ glue together to give a wheel in $R$. (It is closed because each import in one string corresponds to an export in another string.)

1.5.14 Lemma. If the indexing graph $R$ is loopfree, then each of the maps $G_{k} \rightarrow Q$ is an open inclusion.

\subsection{Complements and convexity}

The material in this subsection will only be needed again in Subsection 2.4.

1.6.1 Naive complement. If $H \rightarrow G$ is a subgraph (i.e levelwise injective), then the naive complement is simply defined by taking complements levelwise. It is again a subgraph. This notion is not very useful for the present purposes, where the emphasis in on etale maps. The better notion is the following adjustment.

1.6.2 Etale complement. If $H \rightarrow G$ is a subgraph, we define the etale complement to be the etale hull of the naive complement. We denote it $H^{\complement}$ or $G \backslash H$. Precisely, if temporarily $H^{\prime}$ denotes the naive complement, then $H^{\prime} \rightarrow G$ is injective and in particular locally injective, and we take its core-equivalence/etale factorisation (1.4.4)

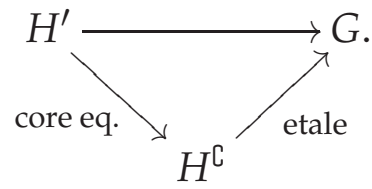

By the colimit formula for etale hull (1.5.9), the complement can also be described as

$$
H^{\complement}=\operatorname{colim}\left(\operatorname{el}\left(H^{\prime}\right) \rightarrow \operatorname{el}(G) \rightarrow \mathbf{G r}^{+}\right) .
$$

1.6.3 Etale complement of open subgraphs. In the special case where $H \rightarrow G$ is an connected open subgraph, which contains at least one node, then $G \backslash H$ is just the open hull of the remaining nodes in $G$. In particular, $H^{\complement}$ is again an open subgraph of $G$ (but not in general connected). The intersection $S:=H \cap H^{\complement}$ is a set of edges, namely the 
ports of $H$ that are not also ports of $G$, and $G$ can be recovered by gluing together $H$ and $H^{\complement}$ along $S$. Precisely, $G$ is naturally the colimit of the gluing datum

$$
S \rightrightarrows H+H^{\complement} .
$$

1.6.4 Etale complement of an edge. We shall also need the very special case where $H$ consists of a single edge of $G$. If $H$ consists of a port of $G$, then $H^{\complement}=G$. If $H$ consists of an inner edge $e$ of $G$, then $G \backslash e$ is the graph obtained from $G$ by cutting that edge. More formally, just as $G$ is the colimit of the canonical gluing datum

$$
O \times_{A} I \rightrightarrows \operatorname{cancov} G
$$

(of all the nodes over all the inner edges), $G \backslash e$ is the colimit of

$$
O \times_{A} I \backslash e \rightrightarrows \operatorname{cancov} G .
$$

Hence there is a natural map $G \backslash e \rightarrow G$ which is bijective on nodes and etale but not an inclusion: the inverse image of $e$ consists of two edges.

1.6.5 Convex open subgraphs. Recall (from 1.2.5) that $L_{k}$ denotes the linear graph with $k \geq 0$ nodes. A path in a graph $G$ is a map $L_{k} \rightarrow G$ (not required etale). An connected open subgraph $H \subset G$ is called convex if the inclusion map is right orthogonal to every inclusion of the ports into a linear graph:

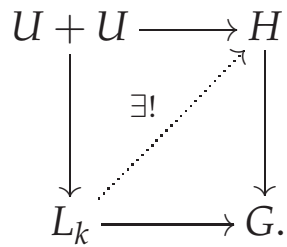

Precisely, $U+U \rightarrow L_{k}$ is import-preserving on the first summand and export-preserving on the second summand. (The horizontal maps and the diagonal filler are not required to be etale.)

1.6.6 Lemma. The composite of two convex inclusions is again convex. The identity map of a connected graph is a convex inclusion. Hence graphs and convex inclusions form a subcategory of $\mathbf{G r}$.

Proof. This is clear since convex inclusions form a right orthogonal class.

1.6.7 Edge poset. If $X$ is a graph, then

$$
I \times{ }_{N} O \rightarrow A \times A
$$

is a relation on $A$, denoted $\lessdot:$ we have $x \lessdot y$ iff there is a node with $x$ as incoming edge and $y$ as outgoing edge. An edge $x$ is a loop iff $x \lessdot x$, so that $X$ is loopfree iff $\lessdot$ is anti-reflexive. The graph is acyclic iff the transitive closure of $\lessdot$ is anti-symmetric and anti-reflexive. 
Assuming that $X$ is acyclic, the transitive and reflexive closure of $\lessdot$, denoted $\leq$, is a poset called the edge poset of $X$. We have $x \leq y$ iff there is a path from $x$ to $y$ in the graph. Hence a connected open subgraph $H \subset X$ is convex iff the edge poset of $H$ is a convex subset of the edge poset of $X$, in the usual sense $(x, y \in H \& x \leq m \leq y \Rightarrow m \in H)$.

1.6.8 Remark. The notion of convexity makes sense of course also for subgraphs not required to be open or not required to be connected, but then a looser notion of path is needed to detect convexity: specifically, 'linear' graphs starting or ending in a node instead of an edge should be allowed

Conversely, it is also possible to formulate the notion of convexity entirely inside the category of etale maps. In this case the notion of path must be replaced by the notion of strip: a strip is the etale hull of a path. In the lifting diagram, instead of just linear graphs $L_{k}$ it is necessary to use all the graphs $P$ such that $P^{\bullet} \simeq L_{k}^{\bullet}$. Indeed, these are precisely the graphs that can appear in the core-equivalence/etale factorisation of a path, as in 1.4.4.

1.6.9 Convexity in terms of etale complements. Let $H$ be a connected open subgraph of an acyclic graph $G$. If $H$ is a single edge, clearly $H$ is convex. So we assume now that $H$ contains at least one node. The intersection $S:=H \cap H^{\complement}$ is a disjoint union of edges. Convexity says that there are no paths starting and ending in $H$ that go through a node of $H^{\complement}$. Such an offending path must necessarily go through an edge in $S$ (we must leave $H$ somewhere), then through at least one node in $H^{\complement}$, and later through another edge in $S$ (we must enter $H$ again somewhere). So equivalently we can say that there is no path in $H^{\complement}$ between two distinct edges of $S$. In particular, we see that convexity of $H$ does not really depend on what is inside $H$, but only on its boundary and its relationship with the complement.

1.6.10 Terminology from Hackney-Robertson-Yau [10]. Two important notions in [10] can be expressed in terms of convexity: Two nodes in a connected graph are closest neighbours [20], when the open hull of the two nodes is (connected and) convex. A node of a connected graph is almost isolated if the complement is (connected and) convex, or if it is the only node in the graph. (The reader is referred to [10] to see how these notions are defined there (without the notion of convexity), and how they are exploited, among other things, to arrive at the notion of subgraph used there (which in the present terminology is the notion of convex open subgraph).)

\section{Properads}

This section owes a lot to Joyal-Kock [11].

Denote by $G r$ the category of connected acyclic graphs with etale maps. From now on we say simply graph for connected acyclic graph. 


\subsection{Digraphical species (coloured bi-collections) and F-graphs}

2.1.1 Digraphical species $=$ coloured bi-collections. A presheaf

$$
\begin{aligned}
F: \mathbf{e l G r} \mathbf{r}^{\mathrm{op}} & \longrightarrow \text { Set } \\
E & \longmapsto F[E]
\end{aligned}
$$

is called a digraphical species or a (coloured) bi-collection. So it is the data of a 'set of colours' $F[U]$, the value on the unit graph, and for each $(m, n)$, a set $F[m, n]$ of operations of biarity $(m, n)$. Each input and output slot in an operation is labelled by a colour, via the maps in elGr from the unit graph to the corollas.

We favour the terminology digraphical species when $F$ conveys the idea of a structure on digraphs, something to decorate digraphs with, while we prefer the terminology bicollection when $F$ serves as the structure underlying (or freely generating) a properad.

A graph $X$ defines a bi-collection by

$$
\begin{aligned}
X: \mathbf{e l G r} \mathbf{r}^{\mathrm{op}} & \longrightarrow \text { Set } \\
E & \longmapsto \operatorname{Hom}^{\mathrm{et}}(E, X) .
\end{aligned}
$$

The category of elements el $(X)$ introduced in 1.5.5 then coincides with the category of elements of this presheaf (see [15], Ch.III, §7).

2.1.2 Grothendieck topology and sheaves. The category Gr has a natural Grothendieck topology in which a cover is a collection of etale maps that are jointly surjective on nodes and on edges. Every graph has a canonical cover $\operatorname{cancov}(X) \rightarrow X$ which is a disjoint union of elementary graphs, cf. 1.4.1. From (3) we get

$$
\operatorname{PrSh}(\mathrm{elGr}) \simeq \operatorname{Sh}(\mathrm{Gr}) .
$$

Via this equivalence, a digraphical species $F$ can be evaluated on any graph, not just on the elementary ones. The formula is:

$$
F[G]=\lim _{E \in \mathrm{el}(G)} F[E] .
$$

(We already know that $G$ is a colimit of its canonical diagram, in the category $G r$ of graphs and etale maps. That $F$ is a sheaf on Gr means precisely that this colimit is sent to a limit, which is the one in the formula.)

2.1.3 Lemma. In the special case where the presheaf $F: \mathbf{e l G r}^{\mathrm{op}} \rightarrow$ Set is 'represented' by a graph $X$, that is, $F[E]=\operatorname{Hom}^{\mathrm{et}}(E, X)$, then as a sheaf $F: G \mathbf{r}^{\mathrm{op}} \rightarrow$ Set it is genuinely represented by $X$ :

$$
F[G]=\operatorname{Hom}^{\mathrm{et}}(G, X) .
$$

Proof.

$$
F[G]=\lim _{E \in \mathrm{el}(G)} F[E]=\lim _{E \in \mathrm{el}(G)} \operatorname{Hom}^{\mathrm{et}}(E, X)=\operatorname{Hom}^{\mathrm{et}}(\operatorname{colim} E, X)=\operatorname{Hom}^{\mathrm{et}}(G, X) .
$$


2.1.4 F-graphs. Every digraphical species $F$ defines a notion of F-graph. They are graphs whose edges are decorated by the colours of $F$, and whose nodes are decorated by the operations of $F$, subject to obvious compatibility conditions. Formally, the category of $F$-graphs is the comma category $G r \downarrow F$ (or $\mathbf{G r}^{+} \downarrow F$ if we allow nonconnected graphs). If for a moment we denote by $T$ the terminal digraphical species, then $T$-graphs are the same thing as graphs, in the following discussion called naked graphs.

All the basic results about graphs hold also for F-graphs. Coequalisers of gluing data exist for $F$-graphs, just as for graphs, now over $F$-shrubs, but the indexing graph is a naked graph, not an F-graph: the residue of an F-graph is not naturally an F-graph, only a naked graph. (For example, $F$ could be the $(2,1)$-species, whose $F$-graphs are binary trees. The residue of a binary tree is of course not in general a binary tree.) Similarly, a graph of $F$-graphs el $(R) \rightarrow \mathbf{G r}^{+} \downarrow F$ admits a colimit in $\mathbf{G r}^{+} \downarrow F$ (obtained by gluing together all the $F$-graphs according to the incidence relations expressed by the naked graph $R$ ).

(Again we see that the indexing graph is not on the same footing as the ingredients of the colimit. There is no natural notion of substituting an F-graph into the node of a naked graph. What does make sense is to use the naked graph as a shape of colimit to compute in the category $\mathbf{G r}^{+} \downarrow F$. It's a recipe for gluing. Hence in the present formalism, gluing is the fundamental notion, while substitution is derived from it.)

\subsection{The free-properad monad}

We shall define properads as algebras for a certain monad. This properad monad was also described by Hackney-Robertson-Yau [10] (Ch.2), and in a more general setting by Yau-Johnson [20] (Ch.10-11), in both cases in terms of graph substitution. The present description of the monad is literally the same as that for coloured modular operads of Joyal-Kock [11], where in turn it is mentioned that it is just the coloured version of the construction of Getzler-Kapranov [9].

2.2.1 $(m, n)$-graphs. An $(m, n)$-graph is a graph $G$ equipped with an isomorphism $\operatorname{res}(G) \simeq C_{n}^{m}$. More formally, the groupoid of $(m, n)$-graphs $(m, n)-G \boldsymbol{r}_{\text {iso }}$ is the homotopy fibre over $C_{n}^{m}$ of the functor res : $G \boldsymbol{r}_{\text {iso }} \rightarrow$ Cor. Note that res : $G r_{\text {iso }} \rightarrow$ Cor is a groupoid fibration, not a discrete fibration, since a graph may well have automorphisms that fix all ports. We are interested in its fibrewise $\pi_{0}$ : that's the essential part of a digraphical species, which to $(m, n)$ assigns $\pi_{0}\left((m, n)-G \boldsymbol{r}_{\text {iso }}\right)$. (It does not say anything about colours, the values on the unit graph, but this information will be provided automatically in the construction below.)

If $F$ is a digraphical species, there is a residue functor res : $G r_{\text {iso }} \downarrow F \rightarrow$ Cor from $F$-graphs to naked corollas. The groupoid of $(m, n)$-F-graphs, denoted $(m, n)-G \mathbf{r}_{\text {iso }} \downarrow F$, is the homotopy fibre over $C_{n}^{m}$ of this functor. 
2.2.2 Underlying endofunctor of the free-properad monad. Let I denote the unit graph. We define the monad for properads:

$$
\begin{aligned}
\operatorname{PrSh}(\text { elGr }) & \longrightarrow \operatorname{PrSh}(\text { elGr }) \\
F & \longmapsto \bar{F},
\end{aligned}
$$

where $\bar{F}$ is the bi-collection given by $\bar{F}[1]:=F[1]$ and

$$
\begin{aligned}
\bar{F}[m, n] & :=\operatorname{colim}_{G \in(m, n)-G r_{\text {iso }}} F[G] \\
& =\sum_{G \in \pi_{0}\left((m, n)-G r_{\text {iso }}\right)} \frac{F[G]}{\operatorname{Aut}_{(m, n)}(G)} \\
& =\pi_{0}\left((m, n)-G r_{\text {iso }} \downarrow F\right) .
\end{aligned}
$$

Here the first equation follows since $(m, n)-G r_{\text {iso }}$ is just a groupoid: the sum is over isomorphism classes of $(m, n)$-graphs, and $\operatorname{Aut}_{(m, n)}(G)$ denotes the automorphism group of $G$ in $(m, n)-G r_{\text {iso }}$.

2.2.3 Multiplication for the monad. $\bar{F}[m, n]$ is the set of isomorphism classes of $(m, n)$ F-graphs: it is the set of ways to decorate $(m, n)$-graphs by the digraphical species $F$. Now $\overline{\bar{F}}[m, n]$ is the set of $(m, n)$-graphs decorated by F-graphs: this means that each node is decorated by an $F$-graph with matching ports. We can use the $(m, n)$-graph as indexing a diagram of F-graphs, and then take the colimit. This describes the monad multiplication

$$
\mu_{F}: \overline{\bar{F}} \rightarrow \bar{F} .
$$

More formally, the groupoid $G r_{\text {iso }} \downarrow \bar{F}$ has as objects pairs $(R, \phi)$ where $R$ is a graph, and $\phi: \operatorname{Hom}(-, R) \rightarrow \bar{F}$ is a natural transformation. Equivalently we can regard $\phi$ as a functor el $(R) \rightarrow \mathbf{e l G} \downarrow \mathbf{r} \downarrow \bar{F}$. Now there is also a canonical functor elGr $\downarrow \vec{F} \rightarrow \mathbf{G r} \downarrow F$, which takes unit graphs to unit graphs, and takes a corolla decorated by an $F$-graph to that same F-graph. The composite functor

$$
\operatorname{el}(R) \rightarrow \mathbf{e l G} \boldsymbol{r} \downarrow \bar{F} \rightarrow \mathbf{G} \boldsymbol{r} \downarrow F
$$

is a graph of $F$-graphs in the technical sense of 1.5.8, and we take its colimit to obtain a single $F$-graph. The whole construction defines a functor

$$
\boldsymbol{G r}_{\text {iso }} \downarrow \bar{F} \rightarrow \mathbf{G r}_{\text {iso }} \downarrow F,
$$

which is compatible with taking residue by Lemma 1.5.12. The fibrewise $\pi_{0}$ of this functor defines the monad multiplication.

2.2.4 Associativity. Associativity asserts that this square commutes:

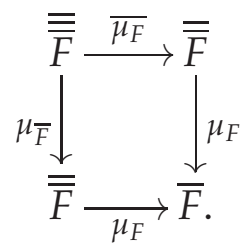


The elements in $\overline{\bar{F}}[m, n]$ are graphs of graphs of $F$-graphs, and associativity amounts to saying that these $F$-graphs can be glued together in two ways with the same result. In detail, an element in $\overline{\bar{F}}[m, n]$ is a graph of $\bar{F}$-graphs, so it amounts to a graph $R$, and for each node $x$ in $R$ an $\bar{F}$-graph $A_{x}$ (and for each inner edge in $R$ the corresponding ports of the $A_{x}$ match). Each $\bar{F}$-graph $A_{x}$ is a graph of $F$-graphs, so for each node in each $A_{x}$ there is an F-graph (and again compatibilities). So altogether there is a number of $F$-graphs involved; associativity says that the following two ways of gluing them all together give the same result. Either inner-first (that's $\mu_{F} \circ \overline{\mu_{F}}$ ): we first glue together, for each $A_{x}$ separately, the corresponding F-graphs, to obtain a set of bigger $F$-graphs indexed by the nodes in $R$, and then finally glue together these bigger $F$ graphs according to the colimit shaped by $R$. Or outer-first (that's $\mu_{F} \circ \mu_{\bar{F}}$ ): we first prepare the overall shape by gluing together all the graphs $A_{x}$ according to the shape $R$. This produces a graph $Q$ of $F$-graphs, and then we use $Q$ as recipe for gluing all the $F$-graphs. Note that there is a natural bijection between the nodes in $Q$ and the sum of all the nodes in all the $A_{x}$ - this follows because the quotient map of the gluing construction $S \rightrightarrows \sum_{x} A_{x} \rightarrow Q$ is bijective on nodes (1.5.2). To see that the two gluing constructions agree, assume first that none of the $A_{x}$ are unit graphs. Start with the outer-first gluing: here we are simply gluing all the $F$-graphs according to one graph $Q$. However, this graph $Q$ contains as open subgraphs all the $A_{x}$. We can perform the colimit construction by first gluing separately over the inner edges of each of the $A_{x}$ (in each case this is a subset of the inner edges in $Q$, and all these subsets are disjoint). But this first step is precisely to assemble all the F-graphs according to which $A_{x}$ they belong to, so it is precisely the first step in the inner-first gluing prescription. Finally we glue along the remaining inner edges in $Q$. By the assumption that none of the $A_{x}$ are unit graphs, these remaining inner edges are precisely identified with the inner edges of the outermost graph $R$. So under this assumption, both ways of gluing are over the same sets of edges. Finally we can easily reduce to this situation from the general case: if there is a node $x$ in $R$ such that $A_{x}$ is a unit graph, then we can start the colimit computation (in either way) by taking the pushout over any inner edge incident to $x$. This pushout does not affect the result, neither the graph $Q$ in the outerfirst calculation, nor the gluing of the $\bar{F}$-graphs $A_{x}$ in the inner-first calculation. We may therefore as well assume that there are no nodes of this type in $R$.

2.2.5 Unit for the monad. The unit for the monad is given by interpreting an $F$-corolla $C \in F[m, n]$ as an $F$-graph. The unit law says: (1) given an $(m, n)$-F-graph $X$, interpreting it first as an $(m, n)$-corolla of $F$-graphs (the single $F$-graph $X$ itself), and then taking the (trivial) colimit, that gives back the $F$-graph $X$ again; and (2), interpreting $X$ as a graph of $F$-corollas, and then taking the colimit of these corollas, also gives back the original F-graph $X$. Both cases are clear.

2.2.6 Properads. A (coloured) properad is defined to be an algebra for the properad monad $F \mapsto \bar{F}$. This means that it is a bi-collection $F:$ elGr ${ }^{\text {op }} \rightarrow$ Set equipped with a structure map $\bar{F} \rightarrow F$ obeying a few easy axioms (cf. [15], Ch. VI): it amounts to a rule which for any $(m, n)$-graph $G$ gives a map $F[G] \rightarrow F[m, n]$, i.e. a way of constructing 
a single operation from a whole graph of them. This rule satisfies some associativity conditions, amounting to independence of the different ways of breaking the computation into steps. Let Prpd denote the category of algebras for the properad monad $F \mapsto \bar{F}$.

2.2.7 Some variations. Polycategories [16], also called dioperads [7], are obtained by using only simply connected graphs (and the same elementary graphs). Operads are obtained by using only rooted trees (and then only elementary graphs that are rooted trees), cf. 1.2.5. See Kock [13]. Categories are obtained by using only linear graphs (and elementary graphs that are linear).

\subsection{Generic/free factorisation and nerve theorem}

This subsection and the next, not really used elsewhere in the paper, introduce and study a bigger category of graphs, whose new maps are generated by the free-properad monad. One important aspect of this bigger category $\widetilde{G} \mathbf{r}$ is the nerve theorem (2.3.9), characterising properads among presheaves on $\widetilde{G} \mathbf{r}$ in terms of a Segal condition. The category $\widetilde{G} \mathbf{r}$ is also described by Hackney-Robertson-Yau [10], although they are more interested in a smaller category (see 2.4.14 below).

2.3.1 Kleisli category. We consider the diagram

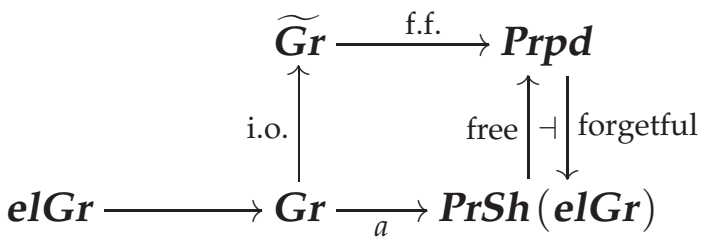

obtained by factoring $\mathbf{G r} \rightarrow \boldsymbol{P r p d}$ as identity-on-objects followed by fully faithful. In other words, $\widetilde{G r}$ is the Kleisli category of the monad (see [15], Ch.VI, §5), restricted to $\boldsymbol{G r}$. This means that a morphism in $\widetilde{G} \boldsymbol{r}$ from graph $R$ to graph $Y$ is defined as a morphism of bi-collections from $R$ to $\bar{Y}$. So where the original maps (those coming from $\mathbf{G r}$, now called free maps) send vertices to vertices, the general maps in $\widetilde{G r}$ send vertices to 'subgraphs' - more precisely, a vertex $x$ of $R$ is sent to an etale map $G_{x} \rightarrow Y$, in both cases subject to compatibility conditions. These conditions say that all the $G_{x}$ form a residue-compatible graph of graphs (2.3.2) indexed by $R$, such that the colimit $Q$ comes with an etale map to $Y$. In particular, in the bigger category $\widetilde{G r}$ there is a new kind of map from $R$ to $Q$ which can be described as refining each of the nodes in $R$, as detailed below. This map realises the construction of 'substituting the graphs $G_{x}$ into the nodes of $R^{\prime}$. The second step in the general map $R \rightarrow Y$ is the etale map $Q \rightarrow Y$. Hence we see that every map in $\widetilde{G} r$ factors as a refinement followed by an etale map. This is an example of generic/free factorisation, an important general phenomenon, and a key ingredient in achieving the nerve theorem below (2.3.9). 
2.3.2 Residue-compatible graphs of graphs. According to 1.5.8, a graph of F-graphs is a functor $\gamma: \operatorname{el}(R) \rightarrow \mathbf{G r} \downarrow F$ that sends all $A$-objects to unit $F$-graphs, and all $I$ maps to import-preserving maps and all $O$-maps to export-preserving maps. We say that a graph of F-graphs $\gamma$ is residue compatible when for each node $x$ in $R$, we have $\operatorname{res}(x)=\operatorname{res}(\gamma(x))$.

2.3.3 Refinements. We first treat the case where the domain is a corolla. To give a map in $\widetilde{G r}$ from $C_{n}^{m}$ to a graph $Y$ is to give a map of presheaves $C_{n}^{m} \rightarrow \bar{Y}$, i.e. an element in $\bar{Y}[m, n]$. By construction this is given by (an isoclass of) a graph $G \in(m, n)-G_{\text {iso }}$ together with an element $[\varphi] \in \operatorname{Hom}(G, Y) / \operatorname{Aut}_{(m, n)}(G)$. We call such a map a refinement if $\varphi$ is invertible. It is now clear that we have the following factorisation into a refinement followed by a free map (i.e. the image of an etale map):

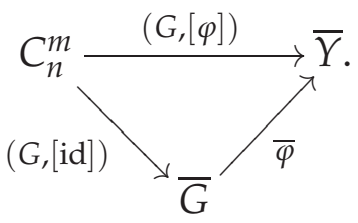

This factorisation is not unique, since each $\sigma \in \operatorname{Aut}_{(m, n)}(G)$ yields a different representative $\varphi \circ \sigma$ for the class $[\varphi]$. But there is clearly a (free) isomorphism between such two factorisations, simply given by $\sigma$ :

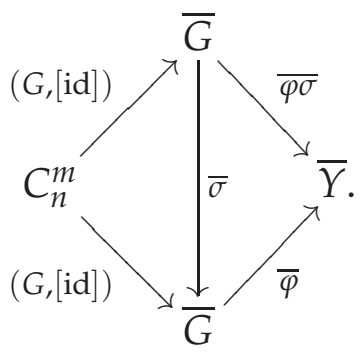

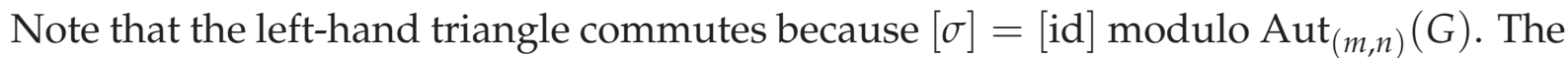
same diagram also shows that the factorisation, although it is unique up to isomorphism, is not in general unique up to unique isomorphism: if $\sigma$ is a nontrivial portpreserving deck transformation of $\varphi$ (this can only happen when there are no ports), then $\varphi=\varphi \sigma$, and the diagram represents a nontrivial automorphism of a factorisation.

The general map in $\widetilde{G r}$, say $R \rightarrow Y$, is essentially a colimit of maps of the previous form. More formally, it is given by a residue-compatible graphs of $Y$-graphs (cf. 2.3.2)

$$
\gamma: \operatorname{el}(R) \rightarrow \mathbf{G r} \downarrow Y .
$$

The colimit of $\gamma$ is a graph $Q$ with an etale map to $Y$. The map $R \rightarrow Y$ is a refinement if this etale map is invertible. In the general case, $R \rightarrow Q \rightarrow Y$ constitutes the refinement/free factorisation. In conclusion:

2.3.4 Proposition. Every map in $\widetilde{G r}$ factors as a refinement followed by a free map. This factorisation is unique up to (non-unique) free isomorphism. 
A version of this factorisation is also obtained in [10] (Lemma 5.43).

2.3.5 Remark. In the preceding discussion, $Y$ was assumed to be a graph, but it fact this is irrelevant: the arguments work exactly the same for $Y$ a general presheaf. In any case a map $R \rightarrow Y$ in the Kleisli category is given by $\gamma: \operatorname{el}(R) \rightarrow \boldsymbol{G r} \downarrow Y$, subject to the same conditions as above, and in any case the middle object $Q$ appearing in the factorisation $R \rightarrow Q \rightarrow Y$ is a graph. This will be important in the proof of the nerve theorem.

2.3.6 Generic maps (cf. [18]). The refinement/etale factorisation is an instance of a very general phenomenon, that of generic/free factorisations and monads with arities, introduced and studied in depth by Weber [18], [19]. A recommended entry point to the theory is Berger-Melliès-Weber [4].

Let $T: \mathscr{C} \rightarrow \mathscr{C}$ be a monad. A (weakly) generic map is a map $g: A \rightarrow T G$ such that for every map $f: X \rightarrow Y$ in $\mathscr{C}$ and every solid square

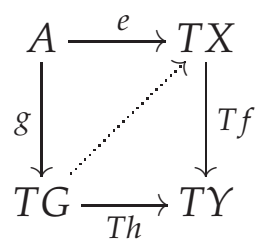

there exists a diagonal filler $d: G \rightarrow X$ (i.e. such that $f \circ d=h$ and $T d \circ g=e$ ). A monad is said to admit (weak) generic factorisations if every map $A \rightarrow T Y$ admits a factorisation as a (weakly) generic map followed by a free map. (Note that we talk about generic maps in the weak sense of [18], not in the strict sense of [19].)

2.3.7 Lemma. The refinement maps are (weakly) generic.

Proof. Given a square as in the definition of generic, with $g$ a refinement map, factor the top map $e$ as refinement followed by free. We now have two different refinement/free factorisations, so by the previous proposition, there exists a free isomorphism comparing them. This provides a (free) filler in the square.

2.3.8 Nerve functor. The embedding $i: \widetilde{G r} \rightarrow \operatorname{Prpd}$ induces the nerve functor

$$
\begin{aligned}
N: \operatorname{Prpd} & \longrightarrow \operatorname{PrSh}(\widetilde{\mathbf{G r}}) \\
X & \longmapsto \operatorname{Hom}_{\text {Prpd }}\left(i\left({ }_{-}\right), X\right)
\end{aligned}
$$

featured in the nerve theorem:

2.3.9 Theorem. The nerve functor $N: \operatorname{Prpd} \rightarrow \operatorname{PrSh}(\widetilde{\boldsymbol{G}} \mathbf{r})$ is fully faithful, and a presheaf is in the essential image of $N$ if and only if it satisfies the Segal condition, i.e. its restriction to $G r$ is a sheaf. 
Proof. It is clear that Gr is small and that $a: G r \rightarrow \operatorname{PrSh}(\mathbf{e l G r})$ is fully faithful and dense. The nerve theorem will be an instance of the general nerve theorem of Weber [19] (Theorem 4.10), if just we can establish that $a: \mathbf{G r} \rightarrow \boldsymbol{P r S h}(\mathbf{e l G r})$ provides arities for the free-properad monad, which temporarily we denote by $T$. By BergerMelliès-Weber [4] (Propositions 2.12-2.14), to say that $a$ provides arities for $T$ is equivalent to saying that the natural functor $a \downarrow T a \downarrow T \rightarrow a \downarrow T$ given by composition has connected fibres. The objects in $a \downarrow T$ are maps $R \rightarrow \bar{Y}$, where $R$ is a graph and $Y$ is an arbitrary presheaf. The fibre over a map $R \rightarrow \bar{Y}$ is the category of factorisations

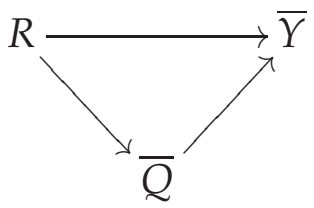

such that the middle object is a graph, and the second map is free. But having (weak) generic factorisation say precisely that this factorisation category has a weakly initial object, and in particular is connected.

2.3.10 Remarks on the proof. Weber established the general nerve theorem ([19], Theorem 4.10) in the situation where $T$ is a monad on a category $\mathscr{C}$ and $a: \Theta_{0} \rightarrow \mathscr{C}$ provides arities for $T$. (To provide arities means that a certain left Kan extension is preserved by the monad.) He showed furthermore ([19], Proposition 4.22) that if $\mathscr{C}$ is a presheaf category and $T$ admits strict generic factorisations, then there is a canonical choice of $\Theta_{0}$, namely the full subcategory spanned by the objects that appear as middle objects of generic/free factorisations of maps from a representable to the terminal presheaf. It was observed in [13] (Remark 2.2.11) that the arguments in Weber's proof in fact yield the more general criterion: $a$ provides arities for $T$ if the natural functor $a \downarrow T a \downarrow T \rightarrow a \downarrow T$ has connected fibres and admits a section. Weber (personal communication) pointed out that in fact the section is not necessary (although of course in practice the section is often provided by generic factorisations). Finally, Berger-MellièsWeber [4] (Propositions 2.12-2.14) turned the criterion into an if-and-only-if statement, and gave a more conceptual formulation and a more elegant proof, as part of a more streamlined overall treatment.

2.3.11 Remarks on weak versus strict generic factorisations. Weber's original notion of generic morphism was the weak notion [18], which is the one relevant in the present work. Subsequent work [19], [13], [4] focused on the strict notion, which is intimately related to the notion of local right adjoint. The weak/strict distinction is closely related with the distinction between analytic and polynomial functors, which in fact was Weber's motivation for introducing the notions of generic map in the first place [18].

Although it is not a precise result at the time of this writing, it seems that in practice the weak situation (related to weakly cartesian monads) always arises from truncation of a strict situation in a homotopical setting, a monad which is cartesian in the homotopical sense. This principle transpires from joint work with David Gepner [8] developing the theory of polynomial functors and generic factorisations in $\infty$-categories, 
and observing in particular that in the $\infty$-world, the difference between analytic and polynomial evaporates. (This and some related results are previewed in [14].)

The present case seems to corrobate this principle. The free-properad monad is only weakly cartesian, due to the presence of the $\pi_{0}$ in the formula for it. In Section 3.3 below, a groupoid-valued version of the monad is described which avoids this truncation. I claim that the groupoid version of the free-properad monad is cartesian and is a local right adjoint, and that it therefore has strict generic factorisations (all in the homotopy sense of [8]).

\subsection{Working in the category $\widetilde{G r}$.}

The category $\widetilde{G r}$ is meant to contain all the combinatorics of graphs relevant to properad theory. The subcategory Gr already has the 'geometric part': open inclusions, etale maps, symmetries, colimits. (In the following when we talk about colimits they are understood to be in Gr.) The new maps introduced, the refinements, represent the algebraic structure, embodying the substitution aspects. It is an important feature of the present approach that this category in which the two aspects interact is generated by general machinery (such as presheaves and monads). While the abstract description as a restricted Kleisli category was enough to establish the generic factorisations and the nerve theorem, it is worthwhile, as we do in this subsection, to extract more explicit descriptions of the refinement maps, and how they interact with the etale maps.

\subsubsection{Lemma. Any map $R \rightarrow Y$ in $\widetilde{\mathbf{G}} \mathbf{r}$ sends edges to edges.}

Proof. Indeed, the map is given by a functor el $(R) \rightarrow \mathbf{G r} \downarrow Y$, assumed to be a residuecompatible graph of graphs in the technical sense of 2.3.2, and in particular it sends $A$-objects of el $(R)$ to unit $Y$-graphs, which is the same as saying that it sends edges to edges.

2.4.2 Hom sets of refinements. Let $R$ and $Y$ be graphs. The set of refinement maps from $R$ to $Y$ is the set of isoclasses of functors el $(R) \rightarrow \mathbf{G r} \downarrow Y$, that are residue-compatible graphs of $Y$-graphs, and with the property that the colimit is terminal. Since all those graphs $G_{x}$ map into $Y$ as open inclusions, instead of calculating the colimit in $G \mathbf{r} \downarrow Y$, we can calculate it in the poset $\operatorname{Sub}(Y)$. Here there are no isomorphisms, so we can say that the set of refinements $R \rightarrow Y$ is the set of residue-compatible graphs of subgraphs-of- $Y$

$$
\{\gamma: \operatorname{el}(R) \rightarrow \operatorname{Sub}(Y) \mid \gamma \operatorname{rcgg}, \operatorname{colim}(\gamma)=\gamma\} .
$$

In the special case where $R=C_{n}^{m}$, the unique node must be sent to the subgraph $Y$ itself, so the only choice involved is where to send the edges, which amounts to specifying an isomorphism $C_{n}^{m} \simeq \operatorname{res}(Y)$. So when this is possible at all ( $Y$ has the correct residue), there are $m ! n$ ! elements in the hom set.

On the other hand, for $Y$ fixed, we can describe the set of all possible refinements $R \rightarrow Y$, with variable $R$. They are given precisely by the bijective-on-nodes open covers of $Y$, i.e. collections of open subgraphs of $Y$ such that each node is in precisely 
one subgraph. Such an open cover is a gluing datum, and $R$ is the indexing graph of it. (This cover interpretation of generic maps was used by Berger [3] in a more general context (see also [13]), and can be seen as a historical precursor to the notion of generic map.)

2.4.3 Lemma. A refinement is completely determined by its values on edges.

Proof. Given a refinement $R \rightarrow Y$, for each node $x$ in $R$ we have the canonical neighbourhood which is a corolla in $R$. We can restrict the refinement to each of these corollas (see 2.4.6 below for details), and in each case, by the previous paragraph, the refinement is determined by its values on edges. Hence also the whole map is determined by its values on edges.

2.4.4 Remark. We also observed (1.1.11) that an etale map is determined by its values on edges, except when the domain has no edges. Even with this exception, this does not imply that a general map in $\widetilde{G r}$ is determined by its value on edges, because the edge map does not determine the factorisation. (For examples, see [10].)

\subsubsection{Composition in $\widetilde{G r}$. Given maps in $\widetilde{G r}$}

$$
R \rightarrow Q \rightarrow Q^{\prime}
$$

where $R \rightarrow Q$ is given by a diagram $\gamma: \operatorname{el}(R) \rightarrow G r \downarrow Q$ sending a node $x$ in $R$ to some etale map $G_{x} \rightarrow Q$, with a chosen isomorphism $\operatorname{res}(x) \simeq \operatorname{res}\left(G_{x}\right)$, and where $Q \rightarrow Q^{\prime}$ is given by a diagram $\delta: \operatorname{el}(Q) \rightarrow G r \downarrow Q^{\prime}$. Then the composite map $R \rightarrow Q^{\prime}$ is described as follows. Put

$$
G_{x}^{\prime}:=\operatorname{colim}\left(\operatorname{el}\left(G_{x}\right) \rightarrow \operatorname{el}(Q) \stackrel{\delta}{\rightarrow} G r \downarrow Q^{\prime}\right)
$$

These graphs are the ingredients of the new diagram $\gamma^{\prime}: R \rightarrow G r \downarrow Q^{\prime}$, which defines the composite map. (Essentially we are just saying that a colimit indexed by a colimit can be expressed as a single colimit, and basically we are just repeating the associativity argument.)

2.4.6 Refactoring etale/refinement as refinement/etale. As a special case, given an etale map $R^{\prime} \rightarrow R$ and a refinement map $R \rightarrow Q$ defined by $\gamma: \operatorname{el}(R) \rightarrow$ Gr (with colimit $Q$ ), then in the diagram

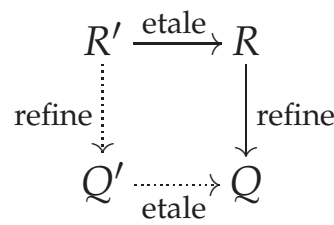

put $Q^{\prime}:=\operatorname{colim}\left(\mathrm{el}\left(R^{\prime}\right) \rightarrow \operatorname{el}(R) \stackrel{\gamma}{\rightarrow} G \boldsymbol{r}\right)$. By construction this defines a refinement $R^{\prime} \rightarrow Q^{\prime}$, and an etale map $Q^{\prime} \rightarrow Q$ is induced from the description of $Q^{\prime}$ as a colimit. 
2.4.7 Proposition. Given an open inclusion $H \rightarrow G$ and a refinement $H \rightarrow Q$, the pushout

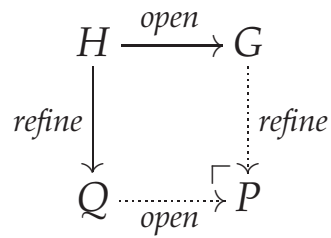

exists in $\widetilde{\mathbf{G r}}$, and the dotted maps are again an open inclusion and a refinement as indicated. The pushout is calculated by identity extension (see proof), and in particular there is a natural isomorphism $P \backslash Q \simeq G \backslash H$.

Proof. If $H \rightarrow Q$ is given by el $(H) \rightarrow$ Gr with colimit $Q$, we need to extend to a functor $\gamma: \operatorname{el}(G) \rightarrow G r$ and define $P$ to be its colimit. Simply assign to each node $x$ in the complement of $H$ the graph given by the canonical neighbourhood of $x$ in $G$. The colimit description provides the etale map $Q \rightarrow P$, and from 2.4.6 it is clear that the resulting square commutes. Alternatively, just as $G$ is obtained by gluing $H$ to its complement $H^{\complement}$ along $S:=H \cap H^{\complement}$, the new graph $P$ is obtained by gluing $Q$ to $H^{\complement}$ along $S$. This makes sense canonically since $S$ is a subset of the set of ports of $H$, and since $Q$ and $H$ have the same ports. From this description, it is clear that $Q \rightarrow P$ is an open inclusion again. It remains to check that the square is a pushout, but again this follows from the construction of $P$ : given another commutative square with the same solid part and with a different $P^{\prime}$ instead of $P$, we need to establish that there is a unique map $P \rightarrow P^{\prime}$ making every everything commute. To give this map is to give el $(P) \rightarrow \mathbf{G} \boldsymbol{r} \downarrow P^{\prime}$, and it is readily seen that there is a unique such functor, since the nodes in $P$ are identified with the nodes in $Q$ plus the nodes in $G \backslash H$.

(Note that it is not true in general that etale maps allow pushouts along refinements.)

2.4.8 Graph substitution (cf. [20], [10].) In the situation of Proposition 2.4.7, if $\mathrm{H}$ consists of a single node $x$, then $Q$ is a graph with $\operatorname{res}(Q)=\operatorname{res}(x)$, and $P$ is the result of substituting $Q$ into the node $x$ of $G$.

2.4.9 Lemma. In the situation of Proposition 2.4.7, if $H \rightarrow G$ is convex, then $Q \rightarrow P$ is convex.

Proof. This follows immediately from $P \backslash Q \simeq G \backslash H$, together with the complement characterisation of convexity (1.6.6).

2.4.10 Corollary. If an open subgraph arises from refinement of a single node, then it is convex.

Conversely:

2.4.11 Lemma. If $Q \subset P$ is a convex open subgraph (of an acyclic graph $P$ ), then there exists an (acyclic) graph $G$ with a node $x$ and a refinement $x \rightarrow Q$ yielding $Q \subset P$ by pushout. 
Proof. If $G$ and $x$ exist, we must have $G \backslash x=P \backslash Q$. Put $S:=Q \cap Q^{\complement}$, then $P$ is the gluing

$$
S \rightrightarrows Q+Q^{\complement} \rightarrow P .
$$

Since $S \subset$ ports $(Q)=$ ports $($ res $Q)$, we can glue in $\operatorname{res}(Q)$ instead of $Q$, obtaining $G$ in this way:

$$
S \rightrightarrows \operatorname{res}(Q)+Q^{\complement} \rightarrow G .
$$

It remains to see that $G$ is acyclic - this is where convexity of $Q$ comes in: a wheel in $G$ through $x$ would induce a path in $G \backslash x=P \backslash Q$ from an edge in $S$ to another edge in $S$. But this is impossible since $Q$ is convex (1.6.6). (And of course $G$ cannot contain a wheel not through $x$, since they would also be a wheel in $G \backslash x=P \backslash Q \subset P$.)

(Note that $G$ might be an inner edge in $Y$; then the complement is not a subgraph.)

Corollary 2.4.10 and Lemma 2.4.11 together are also established in [10], Theorem 5.38, modulo set-up and terminology.

2.4.12 Lemma. In the situation of 2.4.10 and 2.4.11,

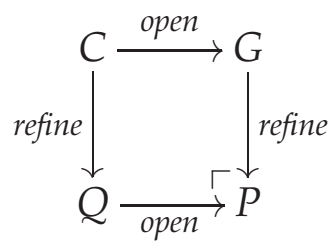

where $C$ is a corolla, suppose $x$ and $y$ are edges in $P \backslash Q=G \backslash C$. If there is a path in $P$ from $x$ to $y$, then there is also a path in $G$ from $x$ to $y$.

Proof. If the path is disjoint from $Q$ it is also a path is $G$. Otherwise, since $Q$ is convex, the path cannot enter and leave $Q$ twice. So it goes in three steps: first from $x$ to $x^{\prime} \in \operatorname{im}(Q)=\operatorname{im}(C)$, second from $x^{\prime}$ to $y^{\prime}$ inside $Q$, and third from $y^{\prime} \in \operatorname{ex}(Q)=\operatorname{ex}(C)$ to $y$. Now there is clearly also a path in $C$ from $x^{\prime}$ to $y^{\prime}$, so by concatenation of paths there is a path in $G$ from $x$ to $x^{\prime}$ to $y^{\prime}$ to $y$.

2.4.13 Proposition. In any commutative square in $\widetilde{\mathbf{G r}}$

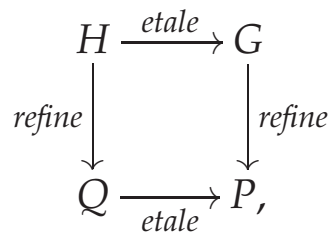

if $H \rightarrow G$ is a convex inclusion then $Q \rightarrow P$ is a convex inclusion.

In other words, refactoring etale/refinement to refinement/etale as in 2.4.6, takes convex/refinement to refinement/convex. 
Proof. The square factors vertically as

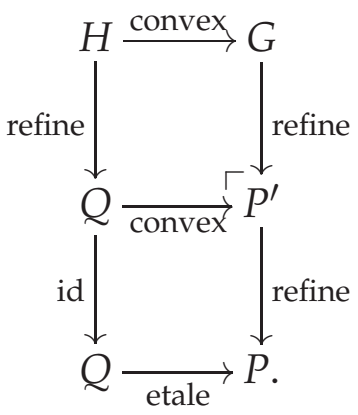

Here the vertical maps in the top square refine nodes in $H$, and the middle map is convex by Proposition 2.4.7. The vertical maps in the bottom square refine nodes outside $Q$. Therefore $Q \rightarrow P$ is an open inclusion since $Q \rightarrow P^{\prime}$ is. Suppose there were a path in $P$ violating convexity of $Q \rightarrow P$. Then by iterated use of Lemma 2.4.12 there would also be a path in $P^{\prime}$ violating the convexity of $Q \rightarrow P^{\prime}$.

2.4.14 Hackney-Robertson-Yau category. Proposition 2.4.13 is essentially equivalent to Lemma 5.50 of [10], modulo set-up and terminology. It follows from the proposition (together with Lemma 1.6.6) that we can obtain a subcategory of $\widetilde{G r}$ by making the following restriction on the maps: allow only maps whose free part is a convex open inclusion. This is the Hackney-Robertson-Yau category $\Gamma$ of connected acyclic graphs [10].

Note that the refinement/convex factorisations that exist in $\Gamma$ by construction are unique up to unique isomorphism, simply because convex open inclusions are monomorphisms. Hence the class of refinements and the class of convex open inclusions form an orthogonal factorisation system in $\Gamma$.

\section{Hypergraphs}

In a nutshell, the idea is this: the free-properad monad applied to a (bi-collection represented by a) graph $X$ is a bi-collection which is not again a graph. Nevertheless, intuitively it should be represented by a diagram

$$
A \longleftarrow \operatorname{et}^{1}(X) \longrightarrow \operatorname{et}(X) \longleftarrow \operatorname{et}_{1}(X) \longrightarrow A \text {, }
$$

where et $(X)$ consists of etale maps from graphs to $X$, and et ${ }^{1}(X)$ (resp. et $1(X)$ ) consists of etale maps to $X$ with a marked import (resp. export). In other words, the monad promotes all 'subgraphs' to being nodes in their own right. With this proliferation of nodes, it is no longer true that an edge is incoming (or outgoing) of at most one node; in other words, the injectivity axiom is violated and the new structure is no longer a graph. The intuition is that it is instead a directed hypergraph. To formalise these ideas, one further ingredient is needed, namely to use groupoids to correctly deal with automorphisms of etale coverings (deck transformations): for the statement 
to be correct we must use groupoid-enriched hypergraphs. Specifically, we need et $(X)$ to be the groupoid of all etale maps to $X$, not just the set of iso-classes of such.

The main result of this section, Theorem 3.4.5, states that the free properad on a hypergraph is again a hypergraph (given by (6)).

\subsection{Discrete hypergraphs}

The theory of hypergraphs is a extensive research topic, with a variety of different applications in computer science. A standard text book on hypergraphs is Berge [2]. For the notion of directed hypergraph, a classical reference is [5]. Here we take a novel approach to directed hypergraphs, englobing naturally the theory of directed graphs above.

3.1.1 Directed hypergraphs. A directed hypergraph is a diagram of sets

$$
A \stackrel{s}{\longleftarrow} I \stackrel{p}{\longrightarrow} N \stackrel{q}{\longleftarrow} O \stackrel{t}{\longrightarrow} A
$$

for which both $I \rightarrow A \times N$ and $O \rightarrow N \times A$ are relations (i.e. are injective maps). The elements in $N$ are called nodes; the elements in $A$ are called hyperedges. A hyperedge connects one set of nodes to another set of nodes. A directed hypergraph can be represented by two incidence matrices between nodes and hyperedges. A directed hypergraph is called loopfree if these two relations are disjoint, i.e. if also $I+O \rightarrow A \times N$ is a relation. (This simply means that a hyperedge cannot contain the same node in its domain and in its codomain.) Loopfree hypergraphs are what are called hypergraphs in [5], where they are encoded as a single signed incidence matrix. For the present purposes it is essential to allow loops.

From now on we simply say hypergraph for directed hypergraph.

A graph is a hypergraph, since a diagram (1) clearly satisfies the conditions: if $s$ and $t$ are themselves injective, clearly the two spans are relations. For the present purposes, a fruitful interpretation of the hypergraph axiom, is that a hypergraph is locally a graph, in the sense that for each node $x$, the maps $I_{x} \rightarrow A$ and $O_{x} \rightarrow A$ are injective. In fact conversely, if for every node $x$ the maps $I_{x} \rightarrow A$ and $O_{x} \rightarrow A$ are injective then $X$ is a hypergraph. Indeed, $I=\sum_{x \in N} I_{x}$ and $A \times N=\sum_{x \in N} A$, and the map $I \rightarrow A \times N$ is just the sum of all the maps $I_{x} \rightarrow A$. Similarly for $O$.

The notion of hypergraph has a self-duality, in the sense that interchanging the role of nodes and hyperedges yields again a hypergraph. However, the study of hypergraphs is biased, so that all notions are geared towards the embedding of graphs inherent in the choice of symbols. With the asymmetry in mind, we define classes of morphisms as follows. A morphism is a diagram like (2); it is etale if the middle squares are pullbacks, and an open inclusion if it is level-wise injective and etale. (Note that according to this definition, etale maps are arity-preserving for nodes, but not necessarily on hyperedges.)

Observe that we have not required the sets to be finite. This is because the free properad on a graph may be an infinite hypergraph (just as the free category on a 
(closed) directed graph may be an infinite category). The relevant finiteness condition is just that $p$ and $q$ be finite maps. These are called hypergraphs of finite type. Henceforth we only consider hypergraphs of finite type.

3.1.2 The core of a hypergraph. The core of a hypergraph $X$, denoted by $X^{\bullet}$, is the (possibly infinite) closed graph $O \times_{A} I \rightrightarrows N$ given by the diagram

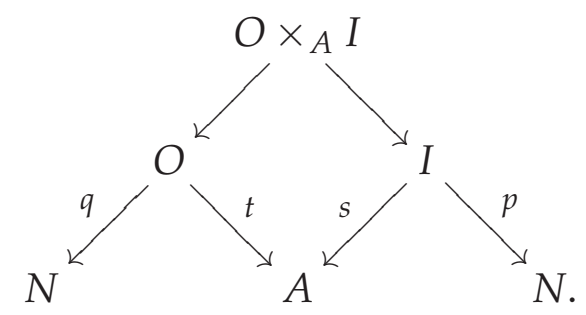

Just as for graphs, we have

3.1.3 Proposition. Taking core is right adjoint to the inclusion of (possibly infinite) closed graphs into hypergraphs.

While for $X$ a graph, the core amounts to deleting all ports, for $X$ a general hypergraph, taking core involves furthermore replacing every hyperedge with a number of edges, one for each connection it realises. The counit is not in general injective.

3.1.4 Inner edges of a hypergraph. The set of inner edges of a hypergraph is by definition the set of edges of its core. Hence the set of inner edges is $O \times_{A} I$. Note that the canonical map $O \times{ }_{A} I \rightarrow A$ from inner edges to hyperedges is not in general injective.

3.1.5 Dual embedding of digraphs. When directed graphs in the classical sense (presheaves on $\bullet \leftleftarrows \bullet$ ) are used as the structures that underlie or generate categories, the nodes play the role of objects (and are not modified by the free-category monad) and the edges generate the arrows. This is in contrast with the free-operad and freeproperad monads, where the edges are left unmodified, and the nodes generate the operations. The contrast is accounted for elegantly by hypergraphs: there is a dual embedding of (possibly infinite) classical directed graphs into hypergraphs, sending $E \rightrightarrows V$ to the hypergraph (of finite type)

$$
V \stackrel{s}{\longleftarrow} E \stackrel{=}{\longrightarrow} E \stackrel{t}{\longrightarrow} V .
$$

In other words, it interprets edges (arrows) as nodes (operations), and interprets vertices as hyperedges.

3.1.6 Proposition. This dual embedding has a right adjoint, sending a hypergraph AINOA to

$$
I \times{ }_{N} O \rightrightarrows A
$$


3.1.7 Sums, connectedness, acyclicity, loops. The notions of connectedness and acyclicity are defined in the same way for hypergraphs as for graphs, but do not play an important role for the present purposes, as the free properad on a connected acyclic graph is a hypergraph which may be neither connected nor acyclic. (Specifically, if a graph $X$ has no ports, then $\bar{X}$ will contain a corresponding isolated node, while each edge in a graph $X$ will become a node in $\bar{X}$ with that same edge as a loop.)

3.1.8 Canonical neighbourhood and canonical cover. The notions of canonical neighbourhood of a node and canonical etale cover are the same for hypergraphs as for graphs: given a subset of nodes $N^{\prime} \subset N$, we construct

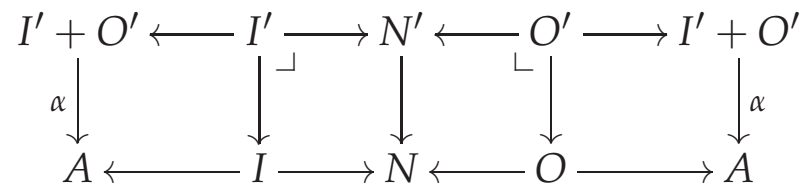

This is clearly a disjoint union of corollas, each of which is the canonical etale neighbourhood of a node in $X$. For $N^{\prime}=N$, these jointly cover $X$. It is the canonical etale cover.

3.1.9 Proposition. The category of hypergraphs and etale maps admits pushouts and coequalisers of shrub injections.

Note that contrary to the case of graphs, there are no import-export conditions: one can glue any hyperedge to any other hyperedge.

Proof. In the presheaf category of diagrams of shape $A I N O A$, pushouts and coequalisers are computed level-wise. It is enough to prove that the results are hypergraphs again. We do pushouts, the case of coequalisers being analogous. Given hypergraphs $A I N O A$ and $A^{\prime} I^{\prime} N^{\prime} O^{\prime} A^{\prime}$, and maps from the shrub S000S, the hyperedge set of the pushout is the amalgamated sum $A+s A^{\prime}$. We need to show that $I+I^{\prime} \rightarrow\left(A+s A^{\prime}\right) \times$ $\left(N+N^{\prime}\right)$ is injective (and similarly with $\left.O+O^{\prime}\right)$. Since the originals are hypergraphs, we have $I+I^{\prime} \hookrightarrow(A \times N)+\left(A^{\prime} \times N^{\prime}\right)$. But we also have $(A \times N)+\left(A^{\prime} \times N^{\prime}\right) \hookrightarrow$ $\left(A+{ }_{S} A^{\prime}\right) \times\left(N+N^{\prime}\right)$ by the distributive law (since $N$ and $N^{\prime}$ are disjoint in $\left.N+N^{\prime}\right)$, as seen in this figure:

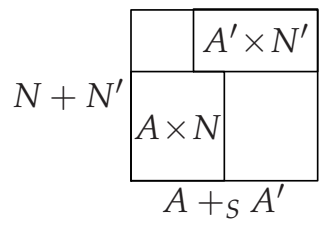

3.1.10 Elements. The category of elements of a hypergraph $X$ is defined exactly as for graphs:

$$
\mathrm{el}(X):=\mathbf{e l G} \boldsymbol{r} \downarrow X,
$$


the category of elements of the presheaf

$$
\begin{aligned}
\mathbf{e l G r}^{\mathrm{op}} & \longrightarrow \text { Set } \\
E & \longmapsto \operatorname{Hom}(E, X) .
\end{aligned}
$$

Just as in the case of graphs, $\mathrm{el}(X)$ is naturally equivalent to the category whose object set is $A+N$ and whose set of non-idenity arrows is $I+O$. Again there is a canonical functor el $(X) \rightarrow \mathbf{G r} \subset \boldsymbol{H G r}$ given by sending each $A$-object to the unit graph $U$, and sending each object $x \in N$ to the canonical neighbourhood of $x$.

3.1.11 Lemma. Every hypergraph $X$ is a colimit of its elements (which are elementary graphs). Precisely,

$$
X=\operatorname{colim}(\mathrm{el}(X) \rightarrow \mathbf{G r} \subset \mathbf{H G r}) .
$$

Proof. The colimit can be computed as an iterated coequaliser over edges.

\subsection{Groupoid-enriched hypergraphs}

3.2.1 Groupoids. We shall freely use basic facts about groupoids, and in particular the consistent homotopy approach. See Gálvez-Kock-Tonks [6], Section 3, where there is some introduction. The important feature is that all notions are up-to-homotopy: in particular, by commutative square is meant a square with a specified 2-cell (a homotopy), and pullback means homotopy pullback. If the bottom corner in a commutative square is just a set, then 'commutative' has its usual meaning and homotopy pullback is the same thing as ordinary pullback. A special case of homotopy pullback is homotopy fibre, which is (homotopy) pullback to a point. We shall also need homotopy quotients (also called action groupoid or semi-direct product), in the situation where a group acts on a set or on a groupoid: where the naive quotient identifies $x$ with $x$.g, the homotopy quotient rather sews in a path between $x$ and $x . g$. The naive quotient is obtained by taking $\pi_{0}$ of the homotopy quotient.

From now on, all pullbacks, fibres, quotients, (and more generally) limits and colimits refer to the homotopy notions.

3.2.2 Groupoid-enriched hypergraphs. A groupoid-enriched hypergraph is a diagram of groupoids

$$
A \stackrel{s}{\longleftarrow} I \stackrel{p}{\longrightarrow} N \stackrel{q}{\longleftarrow} O \stackrel{t}{\longrightarrow} A
$$

satisfying the following three conditions.

1. $A, I$ and $O$ are discrete (i.e. equivalent to sets), and $N$ is locally finite (i.e. has finite vertex groups).

2. $I \rightarrow N \leftarrow O$ are discrete fibrations. (The hypergraph is called of finite type if these fibres are finite. This will always be assumed below.)

3. Both $I \rightarrow A \times N$ and $O \rightarrow N \times A$ are monomorphisms. 
Observe that from condition 1 and 2 we have the diagram<smiles></smiles>

and therefore also $I \rightarrow A \times N$ is automatically a discrete fibration, i.e. has discrete (homotopy) fibres. Condition 3 says that these discrete fibres are either singleton or empty.

Observe that since $I$ (resp. $O$ ) is discrete, and also the fibres $I_{x}$ (resp. $O_{x}$ ) are discrete, the map $I \rightarrow N$ (resp. $O \rightarrow N$ ) is in fact a disjoint union of torsors. More precisely, for each $x \in N$, the vertex group $\operatorname{Aut}_{N}(x)$ acts freely on $I_{x}$ (resp. on $O_{x}$ ). This means that locally at each node $x$, the hypergraph is a 'stacky corolla', as detailed below.

3.2.3 Etale maps. An etale map of groupoid-enriched hypergraphs is a diagram

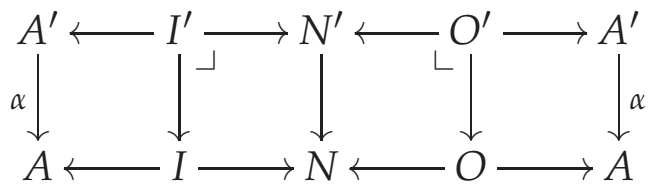

in which the middle squares are (homotopy) pullbacks. We denote by $\mathbf{H G r}$ the category of (groupoid-enriched, finite-type) hypergraphs and etale maps.

3.2.4 Stacky corollas. Consider a corolla $C_{n}^{m}$ :

$$
m+n \leftarrow m \rightarrow 1 \leftarrow n \rightarrow m+n
$$

and suppose that a finite group $G$ acts freely on $m$ and freely on $n$. Then we can form the levelwise homotopy quotient, which receives an etale map from $C_{n}^{m}$ :

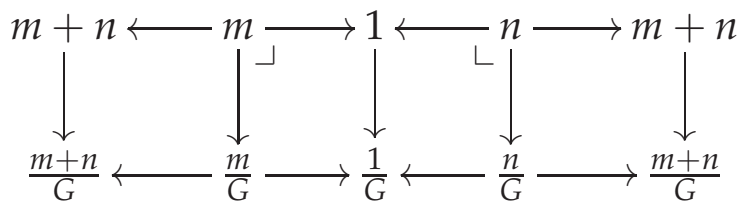

The result is a hypergraph $C_{n}^{m} / G$ called a stacky corolla. It arises as the homotopy coequaliser

$$
C_{n}^{m} \times \underline{G} \rightrightarrows C_{n}^{m} \rightarrow C_{n}^{m} / G,
$$

where $\underline{G}$ denotes the discrete set of elements in the group $G$, and $C_{n}^{m} \times \underline{G}$ denotes the disjoint union of that many copies of $C_{n}^{m}$.

3.2.5 Hypergraphs as colimits of elementary graphs. We need the groupoid version of the result that every hypergraph $X$ is the colimit of its elements. This is true again: the category of elements elGr $\downarrow X$ can be described explicitly as having object set $A+$ 
$\operatorname{obj}(N)$ and arrow set $I+O+\operatorname{arr}(N)$ : an arrow $f \in I$ has domain $s(f)$ and codomain $p(f) \in \operatorname{obj}(N)$, while an arrow $g \in O$ has domain $t(g)$ and codomain $q(g) \in \operatorname{obj}(N)$. The category of elements is the domain of a canonical diagram el $(X) \rightarrow \boldsymbol{H G r}$, whose colimit is $X$. This colimit can be computed as an iterated strict coequaliser of stacky corollas over shrubs, and then the stacky corollas in turn are homotopy quotients as above.

Let $\operatorname{cor}(X):=\operatorname{Cor} \downarrow X$ denote the groupoid of etale maps from corollas into $X$. Let $\operatorname{cor}^{1}(X)$ denote the groupoid of such maps but with a marked import, and let $\operatorname{cor}_{1}(X)$ denote the groupoid of such maps but with a marked export. (Compare 3.4.2.)

3.2.6 Lemma. For X a hypergraph AINOA, there is a natural equivalence of groupoids

$$
\operatorname{cor}(X) \simeq N
$$

Similarly, there are natural equivalences $\operatorname{cor}^{1}(X) \simeq I$ and $\operatorname{cor}_{1}(X) \simeq O$, as well as a bijection hedge $(X):=\operatorname{Map}(U, X) \simeq A$. Altogether, $X$ is equivalent to

$$
\operatorname{hedge}(X) \longleftarrow \operatorname{cor}^{1}(X) \longrightarrow \operatorname{cor}(X) \longleftarrow \operatorname{cor}_{1}(X) \longrightarrow \text { hedge }(X) \text {. }
$$

Proof. Given $x \in N$ we pull back (and use sum injections) to get an etale map

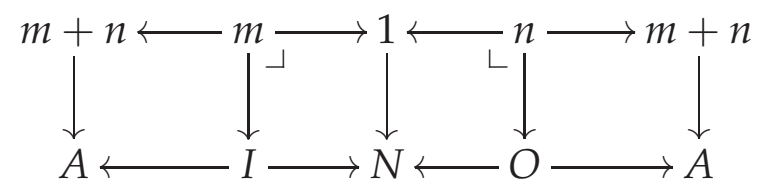

where $m$ is the cardinality of $I_{x}$ and $n$ is the cardinality of $O_{x}$. There are $m$ ! possible bijections $m \stackrel{\sim}{\rightarrow} I_{x}$ and $n$ ! possible bijections $n \stackrel{\sim}{\rightarrow} O_{x}$, but they all yield isomorphic etale maps. The automorphism group of a fixed such map gets no contribution from the $I$ and $O$ level, since these are discrete. The only contribution to automorphisms comes from the automorphisms of $x: 1 \rightarrow N$, and these form precisely the vertex group of $x$. The statements for the remaining sets are straightforward. Note that the first statement is precisely a consequence of the hypergraph axioms. (In fact the condition is equivalent to the hypergraph axioms!)

\subsection{The free properad on a groupoid-valued bi-collection}

The constructions in 2.2 can be carried out with coefficients in groupoids instead of coefficients in sets (and in fact this is in a sense more natural, as we already used groupoids in the constructions, and now avoid taking $\pi_{0}$ in the end). This means that we keep the 1-categories elGr and Gr, but consider presheaves with values in Grpd:

$$
\operatorname{PrSh}(\text { elGr }):=\operatorname{Fun}\left(e^{e l G r}{ }^{\mathrm{op}}, \text { Grpd }\right) .
$$

These could be called prestacks instead of presheaves, as in fact we allow pseudofunctors, but in keeping with the philosophy that the real thing is $\infty$-groupoids, where the 
appropriate weakenings are taken care of automatically by the formalism, and that $\infty$ groupoids are regarded as a fancy version of sets, we stick to the presheaf terminology, and refrain also from going into subtle distinctions between functors and pseudofunctors. As in the set case we have that presheaves (prestacks) on elGr are naturally equivalent to sheaves (stacks) on $\mathbf{G r}$, so that a presheaf on elementary graphs can be evaluated also on general connected graphs by the homotopy limit formula

$$
F[G]=\lim _{E \in \operatorname{el}(G)} F[E] .
$$

Similarly, the definition of the free properad

$$
\begin{aligned}
\operatorname{PrSh}(\text { elGr }) & \longrightarrow \operatorname{PrSh}(\text { elGr }) \\
F & \longmapsto \bar{F},
\end{aligned}
$$

now uses a homotopy colimit:

$$
\begin{aligned}
\bar{F}[m, n] & :=\operatorname{colim}_{G \in(m, n)-G r_{\text {iso }}} F[G] \\
& =\sum_{G \in \pi_{0}\left((m, n)-G r_{\text {iso }}\right)} \frac{F[G]}{\operatorname{Aut}_{(m, n)}(G)} \\
& =(m, n)-G r_{\text {iso }} \downarrow F .
\end{aligned}
$$

Here the double-line denotes the homotopy quotient.

(The resulting functor is a pseudo-monad rather than a strict monad, and the notion of groupoid-enriched properad should be that of pseudo-algebra for this pseudomonad.)

The relationship with the properad monad given in 2.2 is this:

3.3.1 Proposition. The following diagram commutes.

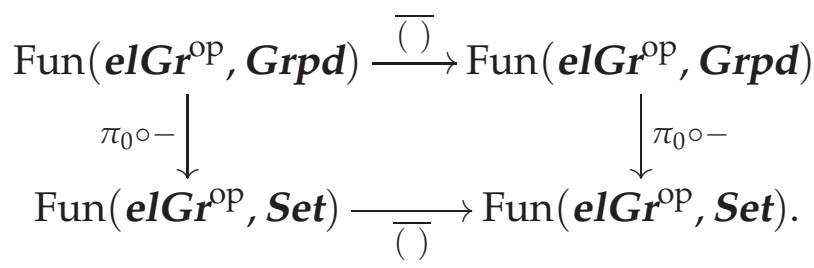

Proof. This follows from the fact that if a group $G$ acts on a groupoid $X$, then there is a natural bijection of sets

$$
\pi_{0}(X / / G) \simeq\left(\pi_{0} X\right) / G,
$$

where the left-hand side is the homotopy quotient and the right-hand side is the naive quotient.

This is particularly interesting if $F$ is the functor given by mapping into a discrete (graph or) hypergraph $X$ : in this case

$$
\operatorname{Map}(E, X)=\pi_{0} \operatorname{Map}(E, X)=\operatorname{Hom}(E, X),
$$

so that the commutativity of the square states that in this case the free-properad monad construction of 2.2 actually factors through the groupoid-enriched version. 


\subsection{Free-properad monad on the category of hypergraphs}

Every hypergraph $X$ defines a bi-collection

$$
\begin{aligned}
X: \mathbf{e l G r} \mathbf{r}^{\mathrm{op}} & \longrightarrow \text { Grpd } \\
E & \longmapsto \operatorname{Map}_{\mathbf{H G r}}(E, X),
\end{aligned}
$$

and can therefore be given as argument to the free-properad monad. The main result of this section states that the free properad on a hypergraph is again a hypergraph. The density lemma 3.2.5 is equivalent to:

3.4.1 Corollary. The natural functor $\mathbf{H G r} \rightarrow \operatorname{PrSh}(\mathbf{e l G r})=\operatorname{Fun}\left(\mathbf{e l G r} \mathbf{r}^{\mathrm{op}}, \mathbf{G r p d}\right)$ is fully faithful.

We give the construction of the free-properad monad purely combinatorially, within the category of hypergraphs, and check that it works. In other words, we define an endofunctor on $\mathbf{H G r}$, and check the commutativity of

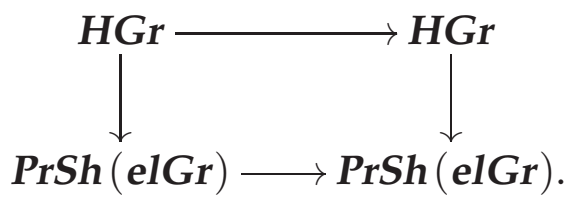

(Since the vertical maps are fully faithful, the top functor acquires monad structure from the bottom functor.)

3.4.2 Free-properad construction for hypergraphs. Given a hypergraph $X$ (with constituents $A I N O A)$, let et $(X)$ denote the groupoid of etale maps from a graph to $X$, i.e. diagrams

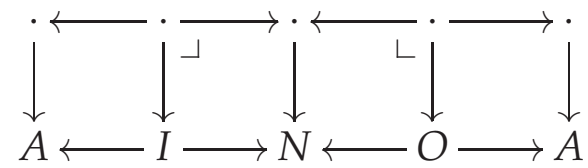

where the first line is a graph.

Let et $^{1}(X)$ denote the groupoid of etale maps from a graph but with a marked import $e$. Formally these are diagrams

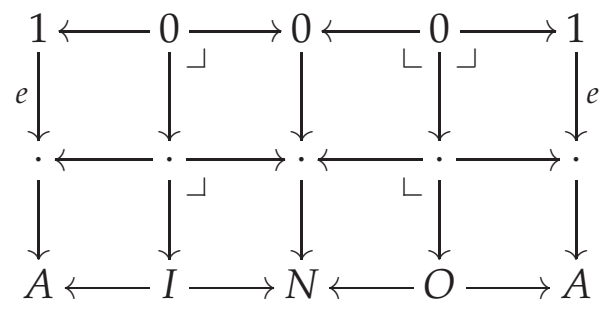

where the middle line is a graph. Similarly, et ${ }_{1}(X)$ is the groupoid etale maps from a graph but with a marked export. 
It is clear that these groupoids assemble into a diagram $\bar{X}$

$$
A \longleftarrow \text { et }^{1} X \longrightarrow \text { et } X \longleftarrow \text { et }_{1} X \longrightarrow A,
$$

where the structure maps delete appropriate rows of the diagrams representing $\operatorname{et}^{1} X$ and et $_{1} X$.

3.4.3 Lemma. If $X$ is a hypergraph, then so is $\bar{X}$, and this assignment is the object part of an endofunctor $\mathbf{H G r} \rightarrow \mathbf{H G r}$.

Proof. We first establish that $\bar{X}$ is a hypergraph. $A$ is discrete by assumption. Let $p$ be a point in et $(X)$, i.e. an etale map $p: G \rightarrow X$, say of degree $d$, and where $G$ is a graph (and in particular is connected). The vertex group $\operatorname{Aut}(p)$ is the group of deck transformations of the covering $p$, and since $G$ is connected, it acts freely on the fibres. In et $^{1}(X)$, there are furthermore marked imports of $G$; the number of imports must be a multiple of $d$. Let $p^{\prime}$ denote the same covering but with a marked import. Any such marked import must be fixed by the vertex group of $p^{\prime}$, and therefore also any adjacent node must be fixed, but since $G$ is connected, it fixes all automorphisms, so $\operatorname{Aut}\left(p^{\prime}\right)$ is trivial. Since $\operatorname{Aut}(p)$ acts freely on the fibres, it also acts freely on the set of imports, hence the homotopy quotient is again discrete, so all the discreteness conditions are satisfied. It remains to establish that et $^{1} X \rightarrow A \times$ et $(X)$ is a monomorphism. So fix a hyperedge $e \in A$ and an etale map $G \rightarrow X$ (element in et $(X)$ ). Well, that hyperedge either is or isn't an import of $G$, so the map is a monomorphism. Similarly of course for exports.

Finally for the functoriality: given an etale map $X \rightarrow Y$, we get maps et $(X) \rightarrow$ et $(Y)$ by postcomposition, and similarly with the markings. These form pullback squares, since for both hypergraphs the $\bar{p}$ and $\bar{q}$ fibres over a subgraph $G$ are the sets of ports of G.

3.4.4 Lemma. Let $X$ be a hypergraph, and let $\bar{X}$ denote the hypergraph constructed in 3.4.2. Then the natural square

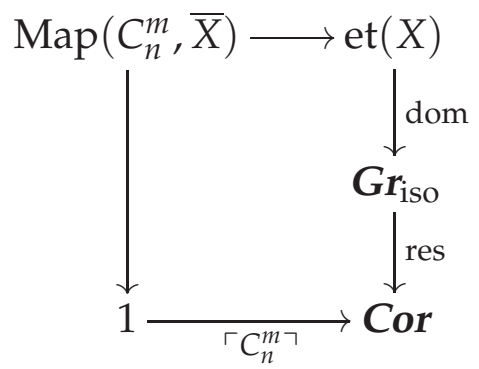

is a (homotopy) pullback. Here res returns the corolla of ports of a graph, and the preceding map sends an etale map $G \rightarrow X$ to its domain.

Proof. An object in this mapping space is a diagram

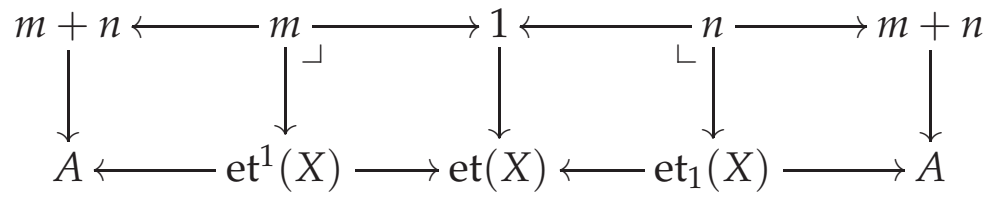


so the main ingredient is to give the middle vertical map, an element in et $(X)$, i.e. an etale map $G \rightarrow X$. The fibre in et $^{1}(X)$ over this element is naturally identified with the set of imports of $G$, so we need next to specify a bijection $m \underset{\rightarrow}{\sim} \operatorname{im}(G)$. Similarly we

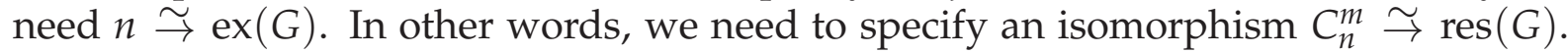
But this is also the description of the pullback. It is easy to see that the arrows in the compared groupoids match up correctly as well.

3.4.5 Theorem. The following diagram commutes.

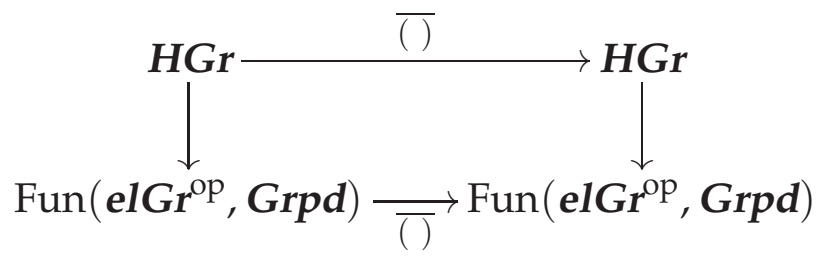

Proof. Let $X$ be a hypergraph. We check that the two presheaves associated to $X$ agree on an elementary graph $C_{n}^{m}$. For this consider the diagram of (homotopy) pullbacks of groupoids:

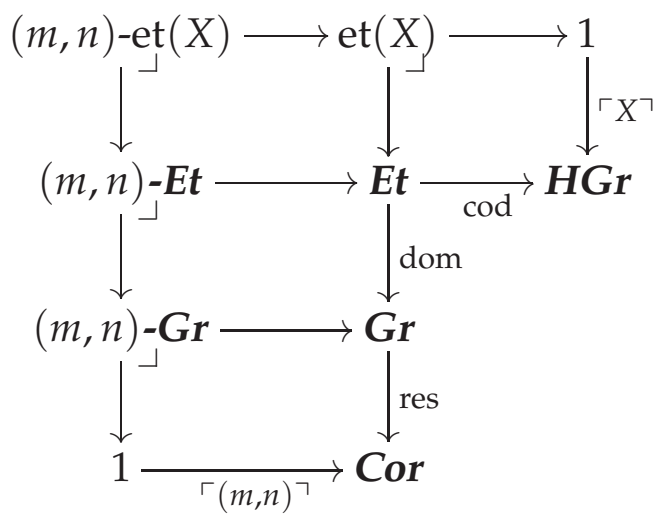

Here we have suppressed notation to indicate that we are talking about groupoids, not categories. Hence HGr denotes the groupoid of hypergraphs, and $\mathbf{G r}$ denotes the groupoid of connected graphs. Furthermore, $\boldsymbol{E t}=\mathbf{G} \boldsymbol{r} \downarrow \mathbf{H G} \mathbf{r}$ denotes the groupoid whose objects are etale maps from a connected graph to a hypergraph, and whose morphisms are pairs of isos in the obvious way. The upper left-hand corner is the groupoid whose objects are data $G \rightarrow X$ together with $(m, n) \stackrel{\sim}{\rightarrow}$ res $G$, consisting of an etale map from a graph into $X$, and a numbering of the ports of $G$. Then the presheaf associated to the hypergraph $\bar{X}$ sends $(m, n)$ to the groupoid $\operatorname{Map}\left(C_{n}^{m}, \bar{X}\right)$. By Lemma 3.4.4, this is precisely the upper left-hand corner of the big diagram. On the other hand, if we apply the free-properad monad to the presheaf associated directly to $X$, the formula for the value on $(m, n)$ is

$$
\sum_{G \in \pi_{0}((m, n)-G r)} \frac{\operatorname{Map}(G, X)}{\operatorname{Aut}_{(m, n)}(G)}
$$

(where the bar now denotes homotopy quotient). But this is precisely the upper lefthand corner of the diagram, expressed as a homotopy-sum of its fibres over objects in $(m, n)-\mathbf{G r}$. 
3.4.6 Remark. Under the 'dual embedding' of (closed) directed graphs into hypergraphs 3.1.5, the free-properad monad restricts to the free-category monad. (In this case, the groupoids involved are discrete.)

\section{References}

[1] Michael Batanin and Clemens Berger. Homotopy theory for algebras over polynomial monads. Preprint, arXiv:1305.0086.

[2] Claude Berge. Hypergraphs, vol. 45 of North-Holland Mathematical Library. NorthHolland Publishing Co., Amsterdam, 1989.

[3] Clemens Berger. A cellular nerve for higher categories. Adv. Math. 169 (2002), 118-175.

[4] Clemens Berger, Paul-André Melliès, and Mark Weber. Monads with arities and their associated theories. J. Pure Appl. Algebra 216 (2012), 2029-2048. ArXiv:1101.3064.

[5] Giorgio Gallo, Giustino Longo, Stefano Pallottino, and Sang Nguyen. Directed hypergraphs and applications. Discrete Appl. Math. 42 (1993), 177-201.

[6] ImMa GÁlveZ-CARrillo, JOACHIM KOCK, and AndreW TONKS. Groupoids and Faà di Bruno formulae for Green functions in bialgebras of trees. Adv. Math. 254 (2014), 79-117. ArXiv:1207.6404.

[7] Wee Liang Gan. Koszul duality for dioperads. Math. Res. Lett. 10 (2003), 109-124. ArXiv:math/0201074.

[8] DAVID GEPNER and JOACHIM KOCK. Polynomial functors over infinity categories. In preparation.

[9] Ezra Getzler and Mikhail M. Kapranov. Modular operads. Compositio Math. 110 (1998), 65-126.

[10] Philip Hackney, Marcy Robertson, and Donald Yau. Infinity Properads and Infinity Wheeled Properads. To appear in Springer Lecture Notes in Mathematics. ArXiv:1410.6716.

[11] ANDRÉ JOYAL and JOACHIM KOCK. Feynman graphs, and nerve theorem for compact symmetric multicategories (extended abstract). In Proceedings of the 6th International Workshop on Quantum Physics and Logic (Oxford 2009), vol. 270 of Electronic Notes in Theoretical Computer Science, pp. 105-113, 2011. ArXiv:0908.2675.

[12] André Joyal and Ieke Moerdijk. A completeness theorem for open maps. Ann. Pure Appl. Logic 70 (1994), 51-86.

[13] JoAchim Kock. Polynomial functors and trees. Internat. Math. Res. Notices 2011 (2011), 609-673. ArXiv:0807.2874.

[14] JOACHIM KOCK. Data types with symmetries and polynomial functors over groupoids. In Proceedings of the 28th Conference on the Mathematical Foundations of Programming Semantics (Bath, 2012), vol. 286 of Electronic Notes in Theoretical Computer Science, pages 351-365, 2012. Arxiv:1210.0828. 
[15] SAUnders MAC LANE. Categories for the working mathematician, second edition. No. 5 in Graduate Texts in Mathematics. Springer-Verlag, New York, 1998.

[16] Manfred E. Szabo. Polycategories. Comm. Algebra 3 (1975), 663-689.

[17] Bruno Vallette. A Koszul duality for PROPs. Trans. Amer. Math. Soc. 359 (2007), 48654943. ArXiv:math/0411542.

[18] MARK WEBER. Generic morphisms, parametric representations and weakly Cartesian monads. Theory Appl. Categ. 13 (2004), 191-234 (electronic).

[19] MARK Weber. Familial 2-functors and parametric right adjoints. Theory Appl. Categ. 18 (2007), 665-732 (electronic).

[20] Donald Yau and Mark W. Johnson. A Foundation for PROPs, Algebras, and Modules. Vol. 203 of Mathematical Surveys and Monographs, American Mathematical Society, Providence, RI. 